\title{
Mathematical Analysis of a Zika Model with reservoirs and Human Movement
}

Kifah Al-Maqrashi ${ }^{1}$, Fatma Al-Musalhi ${ }^{2}$, Ibrahim M. Elmojtaba ${ }^{1}$, Nasser Al-Salti ${ }^{3}$

1 Department of Mathematics, Sultan Qaboos University, Muscat, Oman

2 Center for Preparatory Studies, Sultan Qaboos University, Muscat, Oman

3 Department of Applied Mathematics and Science, National University of Science and Technology, Muscat, Oman

*alsalti@nu.edu.om

\section{Abstract}

A mathematical model for Zika virus is proposed describing the spread of the disease in three interacting populations, namely, human, vector (mosquitoes) and non-human primate (monkeys) inhabiting forests area. Human movement between rural and forest areas has been also considered. It is assumed that Zika virus spreads within non-human primate population, which in turn acts as a reservoir of infection, and then transmitted to the human population through infected mosquitoes. The proposed model incorporates vertical transmission and direct transmission in all populations. The proposed model has been first normalized. The normalized model has been then fully analyzed both qualitatively and quantitatively to investigate the role of the interaction between forest mosquitoes and primates on the ZIKV transmission dynamics. The mathematical analysis includes positivity and boundedness of solutions, derivation of the basic reproduction number $R_{0}$ using the next generation matrix method, sensitivity analysis, existence and stability analysis of all equilibria and bifurcation analysis. Finally, numerical simulations have been carried out to illustrate the obtained theoretical results and to demonstrate the effect of some model parameters in the disease transmission dynamics. The results show that the interaction between forest 
mosquitoes and primates has a significant impact on the ZIKV transmission dynamics among human population through the fraction of susceptible moving to forest areas. Furthermore, the results highlight that the transmission probabilities are as important as the ratios of population size between vector population and human or primate populations in the disease transmission dynamics.

\section{Introduction}

Recently, Zika virus infection has become a perilous danger for the human society 1 . It 2 is a vector borne illness, spreads through the mosquito borne flavi-infection. This infection was first distinguished from rhesus monkeys in 1947 in the Zika forest of Uganda and from people in Nigeria in 1954, yet it did not spread in pestilence structure among the human populace until 2007 [1]. During 2013-2014, the largest epidemic of

Zika virus occurred in Polynesia, France [2]. In 2018, the first principal assembled report of the biggest announced Zika outbreak in India 3]. Although, instances of Zika infection have fallen in the Americas, the Zika infection stays an active threat in certain areas of the world.

The infection is basically spread by the bites of Aedes species mosquitoes. Also, different types of transmission routes have been perceived (e.g., sexually and vertically). Generally, Zika infection causes a non-severe disease, i.e., it was characterized by causing mild symptoms like fever, headache, rash, arthralgia and conjunctivitis. However, a few territories recently influenced by the infection are giving troubling data on the all-around referenced possible relationship of neonatal mutations (microcephaly) and Guillian- Barre syndrome (GBS) with Zika Virus (ZIKV). As an outcome, the WHO pronounced a Public Health Emergency of International Concern on the first of February of 2016, featuring the significance of upgrade the actions to decrease the ZIKV infection, especially among pregnant ladies and ladies of childbearing age 4]. The advancement of Zika immunization will require proceeded with center and speculation. Until a Zika antibody is accessible, counteraction endeavors for pregnant ladies incorporate aversion of movement to territories with dynamic Zika transmission, evasion of mosquito bites for those living in or going to zones with Zika transmission, and insurance against sexual transmission [5].

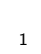

3

4

5

6

7 8 9 
Researchers from different fields are working on understanding the disease transmission dynamics. Mathematical models have been demonstrated as a significant tool to comprehend the disease infection transmission. However, there have been few studies on the role of animals as ZIKV hosts. There are some reports on ZIKV identification in non-human primates which raise the prospect that they could serve as reservoirs. The creation of a sylvatic Zika cycle in the forest of South America is highly likely, according to mathematical models of Zika virus transmission as discussed in [6]. Their model predicts that the inclusion of a quickly reproducing primate or other mammal, that is a competent host for ZIKV, greatly boosts the possibilities of establishing a sylvatic cycle. They suggest that the formation of a ZIKV sylvatic cycle can be supported by a network of as few as 6,000 primates and 10,000 mosquitoes. Furthermore, during the 2011 ZIKV amplification in Kedougou, field researchers revealed that the virus was present in all main land cover classes in the region, but was discovered substantially more frequently in the forest than in other land cover types. Two of the three monkey species found in Kedougou, African green monkeys and Patas monkeys, have previously been found to carry ZIKV. Following that, the virus was discovered in a large swath of tropical Africa using monkey serosurveys and virus isolation from monkeys and many species of sylvatic Aedes. In 7], scientists predict that when monkeys become infected with the sylvatic cycle of Yellow Fever (also a flavivirus) in the Americas, they show overt clinical symptoms and a viremia high enough to transmit virus to mosquito vectors. The virus remained limited to Africa and Asia's tropical areas, infecting monkeys, arboreal mosquitoes, and humans on rare occasions. The majority of primates found to be ZIKV-positive in the wild or in sentinel studies are from the Old World. Humans are more closely linked to Old World primate species, particularly chimps and orangutans, according to phylogenetic study. Hence, the relative risk often increase in diseases that can be transmitted between closely related species. They also discovered that the ZIKV genome sequence in monkeys was identical to the ZIKV circulating in humans in South America, implying that primates sharing the same habitat as humans could serve as ZIKV hosts. In addition, scientists in 8 look into ZIKV infection in wild African monkeys to learn more about its emergence and transmission, and see if there was any signs of active or prior infection. Their findings imply that up to $16 \%$ of some nonhuman primate groups were exposed to

27 
ZIKV at some stage.

Researchers in 9 predict that studying ZIKV in Non-Human Primates (NHP) models can provide insight into viral dynamics as well as serve as a valuable tool for testing antiviral medicines and vaccines. Many of the important clinical findings in human Zika infection have been replicated in NHPs, including quick control of acute viremia, early penetration of the central nervous system, and sustained viral shedding and fetal disease in pregnant animals.

Moreover, in [10], a ZIKV mathematical model incorporating human movement between rural areas and nearby forests was presented to investigate the role of human movement in the spread of Zika virus infections in human and vector populations. The vector compartment, based on mosquito species distributions, have been split into rural areas and proximity forest areas. Authors discussed the consequences of an infected person with mild symptoms moving from rural to nearby forest regions in search of work or sustenance. The effect of a susceptible human mobility on the ZIKV transmission has been also considered. The obtained results showed that the human movement from rural areas to forests has a small effect in increasing the infected human and vector populations.

In this paper, we extend our previous work by proposing a mathematical model that includes three interacting populations, namely, human, mosquitoes (vectors) and monkeys (primates). The vector population has been divided into rural and forest mosquitoes. Direct and vertical transmissions routes are proposed in all populations. The proposed model aims to assess the effect of non-human primate (monkeys) inhabiting forests in spread of ZIKV to the nearby rural areas through the mobility of susceptible humans to forest areas and their interaction with contagious forest mosquitoes. This nearby movement can be happen for different reasons like work or food searching. The rest of this paper is organized as follows: model description is given in Section 2. Mathematical Analysis of the proposed model is discussed in Section 3. The Mathematical analysis includes normalization, positivity and boundedness of solution, basic reproduction number, sensitivity analysis of reproduction number, local and global stability of equilibrium states and bifurcation analysis. Numerical simulations of the proposed model are presented in Section 4, which includes examining the effect of some model parameters variations on the disease transmission dynamics. 


\section{Model Description}

In this section, a model for Zika virus transmission between humans, vectors and

primates (monkeys) is proposed. The interactions between these different populations

are illustrated in Fig 1. The total human population $N_{H}$ is assumed to remain constant and is classified into three compartments: susceptible $S_{h}$, infected $I_{h}$ and recovered $R_{h}$ such that $N_{H}=S_{h}+I_{h}+R_{h}$. Recovered humans are assumed to have a lifelong immunity [2].

Fig 1. Illustrated figure for the interaction between human, vectors and non human primates populations.

The susceptible humans are assumed to gain the infection through bites of infectious female mosquitoes that live in rural areas $I_{v}$ as well as by infectious female mosquitoes that live in forest alienated nearby rural areas $I_{u}$ due to their movement to forest areas. So, we split the vector population according to its living area into: rural population $\left(S_{v},{ }_{101}\right.$ $\left.I_{v}\right)$ and nearby forest population $\left(S_{u}, I_{u}\right)$ with constant total vector populations $N_{V}$ and $N_{U}$ such that $N_{V}=S_{v}+I_{v}$ and $N_{U}=S_{u}+I_{u}$, where $S_{v}$ and $S_{u}$ are the susceptible mosquitoes in rural and nearby forest areas, respectively. Recovery of infected mosquitoes from ZIKV infection is not considered due to its short life span 2 . They are assumed to remain infected for their entire remaining life. 6]. We assume that non-humans primates (reservoirs) are monkeys and are classified into three compartments $S_{p}, I p$ and $R_{p}$ similar to human population with constant total population $N_{P}$ such that $N_{P}=S_{p}+I_{p}+R_{p}$. They live only in forest areas and can get infected only by forest infectious mosquitoes. When monkeys become infected, they present overt clinical signs high enough to transmit virus to the mosquito vectors 7 . Monkeys primates recover at a fixed rate $[6]$ and when challenged again with the virus do not get infected 11. The description of the transmission of the ZIKV infection between these compartments are stated as follows:

(i) Susceptible humans $S_{h}$ can get infected with ZIKV via three main routes [12]: vector transmission by a female mosquito bite, direct transmission via sexual 
transmission or blood transfusion, or vertical transmission by being passed from mother to her newborn child. We assume that a fraction $\epsilon_{1}$ of newborns to infected are affected by Zika. We assume that the Zika-affected newborns enter the infected class. Evidence suggests that this fraction is about 2/3 [13]. A fraction $\kappa$ of the susceptible human individuals progresses from rural areas to the nearby forest area for specific reasons such as work or food searching. So, they may get infection from infectious mosquitoes in forest area as well.

(ii) Susceptible rural mosquitoes $S_{v}$ can only be infected by infectious humans $I_{h}$ and susceptible forest mosquitoes $S_{u}$ can only be infected by infectious primates $I_{p}$. Vertical transmission $\epsilon_{2}, \epsilon_{3}$ of the Zika virus in both vector populations are incorporated, respectively. Evidence suggests that Zika virus is transmitted vertically in the mosquito vector [14] and this is the main pathway it survives the colder months.

(iii) Susceptible primates $S_{p}$ are infected by forest infectious mosquitoes and infectious primates $I_{p}$ can infect forest susceptible mosquitoes $S_{u}$ as well. We assume symptomatic and asymptomatic primates equally infectious to mosquitoes and we lump the two classes in one, $I_{p}$. A proportion $\epsilon_{4}$ of primates population can transmit ZIKV infection vertically. Infectious monkeys can also infect other susceptible partner sexually.

Fig 2. Progression diagram of the of the proposed ZIKV model.

The above description is illustrated in Fig 2 and accordingly, the proposed model is given by the following set of equations: 


$$
\begin{aligned}
S_{h}^{\prime} & =\mu_{H} N_{H}-\mu_{H} \epsilon_{1} I_{h}-\beta_{1} \theta_{1} I_{v} \frac{S_{h}}{N_{H}}-\kappa \beta_{2} \theta_{1} I_{u} \frac{S_{h}}{N_{H}}-\lambda_{1} I_{h} \frac{S_{h}}{N_{H}}-\mu_{H} S_{h} \\
I_{h}^{\prime} & =\mu_{H} \epsilon_{1} I_{h}+\beta_{1} \theta_{1} I_{v} \frac{S_{h}}{N_{H}}+\kappa \beta_{2} \theta_{1} I_{u} \frac{S_{h}}{N_{H}}+\lambda_{1} I_{h} \frac{S_{h}}{N_{H}}-\left(\gamma_{1}+\mu_{H}\right) I_{h} \\
R_{h}^{\prime} & =\gamma_{1} I_{h}-\mu_{H} R_{h} \\
S_{v}^{\prime} & =\mu_{V} N_{V}-\mu_{V} \epsilon_{2} I_{v}-\beta_{1} \theta_{2} S_{v} \frac{I_{h}}{N_{H}}-\mu_{V} S_{v} \\
I_{v}^{\prime} & =\mu_{V} \epsilon_{2} I_{v}+\beta_{1} \theta_{2} S_{v} \frac{I_{h}}{N_{H}}-\mu_{V} I_{v} \\
S_{u}^{\prime} & =\mu_{U} N_{U}-\mu_{U} \epsilon_{3} I_{u}-\beta_{3} \theta_{2} S_{u} \frac{I_{p}}{N_{P}}-\mu_{U} S_{u} \\
I_{u}^{\prime} & =\mu_{U} \epsilon_{3} I_{u}+\beta_{3} \theta_{2} S_{u} \frac{I_{p}}{N_{P}}-\mu_{U} I_{u} \\
S_{p}^{\prime} & =\mu_{P} N_{P}-\mu_{P} \epsilon_{4} I_{p}-\beta_{3} \theta_{1} S_{p} \frac{I_{u}}{N_{P}}-\lambda_{2} S_{p} \frac{I_{p}}{N_{P}}-\mu_{P} S_{p} \\
I_{p}^{\prime} & =\mu_{P} \epsilon_{4} I_{p}+\beta_{3} \theta_{1} S_{p} \frac{I_{u}}{N_{P}}+\lambda_{2} S_{p} \frac{I_{p}}{N_{P}}-\left(\gamma_{2}+\mu_{P}\right) I_{p} \\
R_{p}^{\prime} & =\gamma_{2} I_{p}-\mu_{P} R_{p}
\end{aligned}
$$

\begin{tabular}{|c|c|c|c|}
\hline Symbol & Description & Value(Range) & Source \\
\hline$\mu_{H}$ & Natural death/birth rate of humans & $1 /(60 * 365)$ & Assumed \\
\hline$\mu_{V}$ & Natural death/birth rate of mosquito in rural areas & $1 / 14$ & 15 \\
\hline$\mu_{U}$ & Natural death/birth rate of mosquito in forest areas & $1 / 14$ & 15 \\
\hline$\mu_{P}$ & Natural death/birth rate of primates & $1 /(25 * 365)$ & Assumed \\
\hline$\beta_{1}$ & Biting rate of rural mosquitoes on humans & {$[0.33-1]$} & 1 \\
\hline$\beta_{2}$ & Biting rate of forest mosquitoes on humans & [0.33-1] & $\overline{1}$ \\
\hline$\beta_{3}$ & Biting rate of forest mosquitoes on primates & {$[0.3-0.9]$} & $\overline{6}$ \\
\hline$\theta_{1}$ & Transmission probability of an infection from an infectious mosquito & {$[0.10-0.75]$} & 16 \\
\hline$\theta_{2}$ & Transmission probability of an infection to mosquito & {$[0.30-0.75]$} & $\overline{16}$ \\
\hline$\lambda_{1}$ & Direct (sexual) transmission rate between humans & 0.005 & Assumed \\
\hline$\lambda_{2}$ & Direct (sexual) transmission rate between primates & 0.05 & Assumed \\
\hline$\gamma_{1}$ & Recovery rate of humans & $1 / 6$ & 17 \\
\hline$\gamma_{2}$ & Recovery rate of primates & $1 / 5$ & $\overline{18}$ \\
\hline$\epsilon_{1}$ & Probability of vertical transmission in humans & 0.67 & $\overline{13}$ \\
\hline$\epsilon_{2}$ & Probability of vertical transmission in rural mosquitoes & 0.06 & $\overline{19}$ \\
\hline$\epsilon_{3}$ & Probability of vertical transmission in forest mosquitoes & 0.06 & $\overline{19}$ \\
\hline$\epsilon_{4}$ & Probability of vertical transmission in primates & 0.6 & Assumed \\
\hline$\kappa$ & Fraction of susceptible humans moving from rural to forest areas & 0.3 & Assumed \\
\hline
\end{tabular}

subjected to non-negative initial conditions

$$
\left(S_{h}(0), I_{h}(0), R_{h}(0), S_{v}(0), I_{v}(0), S_{u}(0), I_{u}(0), S_{p}(0), I_{p}(0), R_{p}(0)\right)^{T}
$$

The parameter values of the model (1) is given in Table 1 .

Table 1. Description of the constant parameters used in model (1) and its values (per day).

Model Mathematical Analysis In this section, we first normalize the proposed model 
11. Then, we study positivity of solutions and invariant set, the basic reproduction

number and its sensitivity analysis. Existence of equilibrium points, their local and global stability and bifurcation analysis are also considered.

\section{Model Normalization}

To normalize the proposed model [1], let

$$
\begin{aligned}
& S_{H}=\frac{S_{h}}{N_{H}}, \quad I_{H}=\frac{I_{h}}{N_{H}}, \quad R_{H}=\frac{R_{h}}{N_{H}}, \quad S_{V}=\frac{S_{v}}{N_{V}}, \quad I_{V}=\frac{I_{v}}{N_{V}}, \\
& S_{U}=\frac{S_{u}}{N_{U}}, \quad I_{U}=\frac{I_{u}}{N_{U}}, \quad S_{P}=\frac{S_{p}}{N_{P}}, \quad I_{P}=\frac{I_{p}}{N_{P}}, \quad R_{P}=\frac{R_{p}}{N_{P}},
\end{aligned}
$$

such that

$$
S_{H}+I_{H}+R_{H}=1, \quad S_{V}+I_{V}=1, \quad S_{U}+I_{U}=1, \quad S_{P}+I_{P}+R_{P}=1 .
$$

Thus, the normalized model is given by:

$$
\begin{aligned}
S_{H}^{\prime} & =\mu_{H}-\mu_{H} \epsilon_{1} I_{H}-\beta_{1} \theta_{1} \alpha_{1} I_{V} S_{H}-\kappa \beta_{2} \theta_{1} \alpha_{2} I_{U} S_{H}-\lambda_{1} I_{H} S_{H}-\mu_{H} S_{H} \\
I_{H}^{\prime} & =\mu_{H} \epsilon_{1} I_{H}+\beta_{1} \theta_{1} \alpha_{1} I_{V} S_{H}+\kappa \beta_{2} \theta_{1} \alpha_{2} I_{U} S_{H}+\lambda_{1} I_{H} S_{H}-\left(\gamma_{1}+\mu_{H}\right) I_{H} \\
R_{H}^{\prime} & =\gamma_{1} I_{H}-\mu_{H} R_{H} \\
S_{V}^{\prime} & =\mu_{V}-\mu_{V} \epsilon_{2} I_{V}-\beta_{1} \theta_{2} S_{V} I_{H}-\mu_{V} S_{V} \\
I_{V}^{\prime} & =\mu_{V} \epsilon_{2} I_{V}+\beta_{1} \theta_{2} S_{V} I_{H}-\mu_{V} I_{V} \\
S_{U}^{\prime} & =\mu_{U}-\mu_{U} \epsilon_{3} I_{U}-\beta_{3} \theta_{2} S_{U} I_{P}-\mu_{U} S_{U} \\
I_{U}^{\prime} & =\mu_{U} \epsilon_{3} I_{U}+\beta_{3} \theta_{2} S_{U} I_{P}-\mu_{U} I_{U} \\
S_{P}^{\prime} & =\mu_{P}-\mu_{P} \epsilon_{4} I_{P}-\beta_{3} \theta_{1} \alpha_{3} S_{P} I_{U}-\lambda_{2} S_{P} I_{P}-\mu_{P} S_{P} \\
I_{P}^{\prime} & =\mu_{P} \epsilon_{4} I_{P}+\beta_{3} \theta_{1} \alpha_{3} S_{P} I_{U}+\lambda_{2} S_{P} I_{P}-\left(\gamma_{2}+\mu_{P}\right) I_{P} \\
R_{P}^{\prime} & =\gamma_{2} I_{P}-\mu_{P} R_{P}
\end{aligned}
$$


where $\alpha_{1}=\frac{N_{V}}{N_{H}}, \alpha_{2}=\frac{N_{U}}{N_{H}}$ and $\alpha_{3}=\frac{N_{U}}{N_{P}}$ with non negative initial condition

$$
X(0):=\left(S_{H}(0), I_{H}(0), R_{H}(0), S_{V}(0), I_{V}(0), S_{U}(0), I_{U}(0), S_{P}(0), I_{P}(0), R_{P}(0)\right)^{T} .
$$

\section{Positivity of Solutions and Positively Invariant Set}

The positivity and boundedness of the state variables are demonstrated in the following theorem :

Theorem 1 The solution

$$
X(t)=\left(S_{H}(t), I_{H}(t), R_{H}(t), S_{V}(t), I_{V}(t), S_{U}(t), I_{U}(t), S_{P}(t), I_{P}(t), R_{P}(t)\right)^{T}
$$

of system (2) with non-negative initial condition $X(0)$ remains positive for all time $t>0$ in a positively invariant closed set

$\Omega:=\left\{X(t) \in R_{+}^{10}: 0 \leqslant S_{H}(t), I_{H}(t), R_{H}(t), S_{V}(t), I_{V}(t), S_{U}(t), I_{U}(t), S_{P}(t), I_{P}(t), R_{P}(t) \leqslant 1\right\}$.

\section{proof}

Assume that the system (2) has non-negative initial condition $X(0)$. Let

$$
S_{H}^{\prime}=\mu_{H}\left(1-\epsilon_{1}\right) I_{H}+\mu_{H} \epsilon_{1} R_{H}-m_{1} S_{H}-\mu_{H}\left(1-\epsilon_{1}\right) S_{H}
$$

where, $m_{1}=\beta_{1} \theta_{1} \alpha_{1} I_{V}+\kappa \beta_{2} \theta_{1} \alpha_{2} I_{U}+\lambda_{1} I_{H}>0$. It follows that

$$
S_{H}^{\prime}+\left[\mu_{H}\left(1-\epsilon_{1}\right)+m_{1}\right] S_{H}=\mu_{H}\left(1-\epsilon_{1}\right)+\mu_{H} \epsilon_{1} R_{H}
$$

then integrating both sides over $(0, \tau)$, we get

$$
\begin{aligned}
S_{H}(\tau)= & {\left[S_{H}(0)+\mu_{H} \int_{0}^{\tau}\left(\left(1-\epsilon_{1}\right)+\epsilon_{1} R_{H}(t)\right) e^{\mu_{H}\left(1-\epsilon_{1}\right) t+\int_{0}^{t} m_{1}(w) d w} d t\right] } \\
& \times e^{-\left(\mu_{H}\left(1-\epsilon_{1}\right) \tau+\int_{0}^{\tau} m_{1}(w) d w\right)} .
\end{aligned}
$$

Clearly, $S_{H}(\tau)$ is positive since $S_{H}(0) \geq 0$. Similar calculations can be done for $I_{H}^{\prime}$ and 
$R_{H}^{\prime}$, we get

$$
\begin{aligned}
I_{H}(\tau) & =\left[I_{H}(0)+\left(\int_{0}^{\tau} \beta_{1} \theta_{1} \alpha_{1} I_{V}(t)+\kappa \beta_{2} \theta_{1} \alpha_{2} I_{U}(t)\right) e^{\left(\gamma_{1}+\mu_{H}\left(1-\epsilon_{1}\right)\right) t-\int_{0}^{t} \lambda S_{H}(u) d u} d t\right] \\
& \times e^{-\left(\gamma_{1}+\mu_{H}\left(1-\epsilon_{1}\right)\right) \tau+\int_{0}^{\tau} \lambda S_{H}(u) d u}>0,
\end{aligned}
$$

and

$$
R_{H}(\tau)=\left[R_{H}(0)+\left(\gamma_{1} \int_{0}^{\tau} I_{H}(t) e^{\mu_{H} t} d t\right)\right] e^{-\mu_{H} \tau}>0
$$

since initial conditions are non-negative. Similarly, one can show that the remaining components of $X(t)$ are positive at $\tau$. Using continuity of solution and $X(0) \geq 0$, we conclude that $\tau$ can not be supremum and hence, solution will remain positive for all $t>0$

Now, assume that $\Psi(t)=\left(\Psi_{1}(t), \Psi_{2}(t), \Psi_{3}(t), \Psi_{4}\right)^{T}$, where,

$$
\left[\begin{array}{c}
\Psi_{1}(t) \\
\Psi_{2}(t) \\
\Psi_{3}(t) \\
\Psi_{4}(t)
\end{array}\right]=\left[\begin{array}{c}
S_{H}(t)+I_{H}(t)+R_{H}(t) \\
S_{V}(t)+I_{V}(t) \\
S_{U}(t)+I_{U}(t) \\
S_{P}(t)+I_{P}(t)+R_{P}(t)
\end{array}\right]
$$

then, we have

$$
\Psi^{\prime}(t)=\left[\begin{array}{c}
\mu_{H}-\mu_{H} \Psi_{1}(t) \\
\mu_{V}-\mu_{V} \Psi_{2}(t) \\
\mu_{U}-\mu_{U} \Psi_{3}(t) \\
\mu_{P}-\mu_{P} \Psi_{4}(t)
\end{array}\right]
$$

Now, solving for each component, we get :

$$
\left[\begin{array}{c}
\Psi_{1}(t) \\
\Psi_{2}(t) \\
\Psi_{3}(t) \\
\Psi_{4}(t)
\end{array}\right]=\left[\begin{array}{c}
1-\left(1-\Psi_{1}(0)\right) e^{-\mu_{H} t} \\
1-\left(1-\Psi_{2}(0)\right) e^{-\mu_{V} t} \\
1-\left(1-\Psi_{3}(0)\right) e^{-\mu_{U} t} \\
1-\left(1-\Psi_{4}(0)\right) e^{-\mu_{P} t}
\end{array}\right]
$$

where $\Psi_{1}(0)=S_{H}(0)+I_{H}(0)+R_{H}(0), \Psi_{2}(0)=S_{V}(0)+I_{V}(0), \Psi_{3}(0)=S_{U}(0)+I_{U}(0)$, 
and $\Psi_{4}(0)=S_{P}(0)+I_{P}(0)+R_{P}(0)$. It is straight forward to say that

$$
\Psi_{i}(t) \leqslant 1 \quad \text { if } \quad \Psi_{i}(0) \leqslant 1, \quad i=1,2,3,4 .
$$

Thus, the set $\Omega$ is positively invariant set since $0 \leqslant \Psi(t) \leqslant 1$. Moreover, if $\Psi(0)>1 \quad{ }_{160}$ then $\lim _{t \rightarrow \infty} \Psi(t)=1$ and therefore the set $\Omega$ is a globally attractive set.

\section{The Basic Reproduction Number}

The model (2) has three different types of equilibria: disease free equilibrium

$\left(D F E: \mathcal{Z}^{0}\right)$, axial equilibrium $\left(A E: \mathcal{Z}^{(1)}\right)$, describes an endemic in populations living in rural area only and endemic equilibrium $\left(E E: \mathcal{Z}^{(2)}\right)$, describes an endemic in both ${ }_{165}$ forest and rural areas. The endemic and axial equilibria will be discussed later.

The disease free equilibrium always exists and is given by

$$
\mathcal{Z}^{0}:=\left(S_{H}^{0}, 0,0, S_{V}^{0}, 0, S_{U}^{0}, 0, S_{P}^{0}, 0,0\right)
$$

where, $S_{H}^{0}=S_{V}^{0}=S_{U}^{0}=S_{P}^{0}=1$. The threshold quantity $\mathcal{R}_{0}$ is defined as the average number of secondary infections generated by one case in a completely susceptible population. It is calculated by well known Next Generation Method [20]. The Jacobian of the transmission matrix is:

$$
\Sigma=\left[\begin{array}{cccc}
\lambda_{1} & \alpha_{1} \beta_{1} \theta_{1} & \kappa \alpha_{2} \beta_{2} \theta_{1} & 0 \\
\beta_{1} \theta_{2} & 0 & 0 & 0 \\
0 & 0 & 0 & \beta_{3} \theta_{2} \\
0 & 0 & \alpha_{3} \beta_{3} \theta_{1} & \lambda_{2}
\end{array}\right]
$$

and Jacobian of the transition matrix is

$$
Q=\left[\begin{array}{cccc}
\mu_{H}\left(1-\epsilon_{1}\right)+\gamma_{1} & 0 & 0 & 0 \\
0 & \mu_{V}\left(1-\epsilon_{2}\right) & 0 & 0 \\
0 & 0 & \mu_{U}\left(1-\epsilon_{3}\right) & 0 \\
0 & 0 & 0 & \mu_{P}\left(1-\epsilon_{4}\right)+\gamma_{2}
\end{array}\right]
$$

then, the next generation matrix $\Sigma Q^{-1}$ is 


$$
\Sigma Q^{-1}=\left[\begin{array}{cccc}
\frac{\lambda_{1}}{\mu_{H}\left(1-\epsilon_{1}\right)+\gamma_{1}} & \frac{\alpha_{1} \beta_{1} \theta_{1}}{\mu_{V}\left(1-\epsilon_{2}\right)} & \frac{\kappa \alpha_{2} \beta_{2} \theta_{1}}{\mu_{U}\left(1-\epsilon_{3}\right)} & 0 \\
\frac{\beta_{1}}{\mu_{H}\left(1-\epsilon_{1}\right)+\gamma_{1}} & 0 & 0 & 0 \\
0 & 0 & 0 & \frac{\beta_{3} \theta_{2}}{\mu_{P}\left(1-\epsilon_{4}\right)+\gamma_{2}} \\
0 & 0 & \frac{\alpha_{3} \beta_{3} \theta_{1}}{\mu_{U}\left(1-\epsilon_{3}\right)} & \frac{\lambda_{2}}{\mu_{P}\left(1-\epsilon_{4}\right)+\gamma_{2}}
\end{array}\right] .
$$

The basic reproduction number $\mathcal{R}_{0}$ is the spectral radius of $\Sigma Q^{-1}$ which can be expressed as:

$$
\mathcal{R}_{0}:=\max \left\{\mathcal{R}_{1}, \mathcal{R}_{2}\right\}
$$

where

$$
\mathcal{R}_{1}=\frac{1}{2}\left[R_{H H}+\sqrt{R_{H H}^{2}+4 R_{H V}}\right], \quad \mathcal{R}_{2}=\frac{1}{2}\left[R_{P P}+\sqrt{R_{P P}^{2}+4 R_{P U}}\right]
$$

$$
\begin{array}{ccc}
\text { with } & \mathcal{R}_{H H}=\frac{\lambda_{1}}{\gamma_{1}+\mu_{H}\left(1-\epsilon_{1}\right)}, \quad \mathcal{R}_{H V}=\frac{\alpha_{1} \beta_{1}^{2} \theta_{1} \theta_{2}}{\mu_{V}\left(1-\epsilon_{2}\right)\left[\gamma_{1}+\mu_{H}\left(1-\epsilon_{1}\right)\right]}, \\
\text { and } \quad \mathcal{R}_{P P}=\frac{\lambda_{2}}{\gamma_{2}+\mu_{P}\left(1-\epsilon_{4}\right)}, & \mathcal{R}_{P U}=\frac{\alpha_{3} \beta_{3}^{2} \theta_{1} \theta_{2}}{\mu_{U}\left(1-\epsilon_{3}\right)\left[\gamma_{2}+\mu_{P}\left(1-\epsilon_{4}\right)\right]} .
\end{array}
$$

It is clear that $\mathcal{R}_{H H}$ and $\mathcal{R}_{P P}$ represent the transmission due to interaction between human population and between primates population, respectively. $\mathcal{R}_{H V}$ and $\mathcal{R}_{P U}$ represent the transmission due to interaction between human and vector in rural areas and interaction between primates and vector in forest areas, respectively. Moreover, the disease threshold occurs at $\mathcal{R}_{0}=1$ which implies either $\mathcal{R}_{1}=1 \quad$ whenever $\quad \mathcal{R}_{H H}+\mathcal{R}_{H V}=1 \quad$ or $\quad \mathcal{R}_{2}=1 \quad$ whenever $\quad \mathcal{R}_{P P}+\mathcal{R}_{P U}=1$. Also, it can be easily proven that the disease infection will die out at $\mathcal{R}_{0}<1$ which implies that $\mathcal{R}_{H H}+\mathcal{R}_{H V}<1$ and $\mathcal{R}_{P P}+\mathcal{R}_{P U}<1$. This will happen by reducing all routes of transmission, $\mathcal{R}_{H H}, \mathcal{R}_{H V} \mathcal{R}_{P P}$, and $\mathcal{R}_{P U}$. However, the disease infection persists in all populations when $\mathcal{R}_{0}>1$, whenever $\mathcal{R}_{H H}+\mathcal{R}_{H V}>1$ and $\mathcal{R}_{P P}+\mathcal{R}_{P U}>1$.

\section{Sensitivity Analysis of the Basic Reproduction Number.}

In this section, sensitivity analysis of the basic reproduction number $\mathcal{R}_{0}$ is discussed. It 190 allows us to determine the parameters which have significant impact on threshold ratio 191 
$\mathcal{R}_{0}$ of model (2), i.e, a small change in a highly sensitive parameter leads to a high quantitative variation in $\mathcal{R}_{0}$ and may produce qualitatively different results. Such parameters must deserve the attention of management and control strategies. Here, the normalized forward sensitivity index method (elasticity index) 21] is used and it is defined as the ratio of the relative change of $\mathcal{R}_{0}$ with respect to relative variation in a parameter $\ell$ as follows

$$
\Upsilon_{\ell}^{\mathcal{R}_{0}}=\frac{\ell}{\mathcal{R}_{0}} \times \frac{\partial \mathcal{R}_{0}}{\partial \ell}
$$

Since $\mathcal{R}_{0}:=\max \left\{\mathcal{R}_{1}, \mathcal{R}_{2}\right\}$, the sensitivity analysis of $\mathcal{R}_{0}$ with respect to each of its parameter will be evaluated via the sensitivities of each of $\mathcal{R}_{1}$ and $\mathcal{R}_{2}$ such that

$$
\Upsilon_{\ell_{1}}^{\mathcal{R}_{1}}=\frac{\ell_{1}}{\mathcal{R}_{1}} \times \frac{\partial \mathcal{R}_{1}}{\partial \ell_{1}}, \quad \Upsilon_{\ell_{2}}^{\mathcal{R}_{2}}=\frac{\ell_{2}}{\mathcal{R}_{2}} \times \frac{\partial \mathcal{R}_{2}}{\partial \ell_{2}}
$$

where $\ell_{1}, \ell_{2}$ denotes the parameter related to $\mathcal{R}_{1}$ and $\mathcal{R}_{2}$, respectively. Using the explicit expression of the basic reproduction number $\mathcal{R}_{0}(5)$ with the base line values of parameters listed in Table 1, the estimated values of the sensitivities indices at $\mathcal{R}_{0}>1$ are listed in Table 2

Table 2. Sensitivity indices of $\mathcal{R}_{1}$ and $\mathcal{R}_{2}$.

\begin{tabular}{|c|c|c||c|c|c|}
\hline \multicolumn{2}{c}{$\mathcal{R}_{0}=\mathcal{R}_{1}>\mathcal{R}_{2}>1$} & \multicolumn{3}{c}{$\mathcal{R}_{0}=\mathcal{R}_{2}>\mathcal{R}_{1}>1$} \\
\hline Parameter $\left(\ell_{1}\right)$ & Sign & $\Upsilon_{\ell_{1}}^{\mathcal{R}_{01}}$ & Parameter $\left(\ell_{2}\right)$ & Sign & $\Upsilon_{\ell_{2}}^{\mathcal{R}_{02}}$ \\
\hline$\beta_{1}$ & + & 0.9889 & $\beta_{3}$ & + & 0.8775 \\
$\lambda_{1}$ & + & 0.0111 & $\lambda_{2}$ & + & 0.1224 \\
$\gamma_{1}$ & - & 0.5055 & $\gamma_{2}$ & - & 0.5611 \\
$\alpha_{1}$ & + & 0.4944 & $\alpha_{3}$ & + & 0.4387 \\
$\mu_{H}$ & - & $0.4513 \mathrm{e}-4$ & $\mu_{P}$ & - & 0.0001234 \\
$\mu_{V}$ & - & 0.4944 & $\mu_{U}$ & - & 0.4387 \\
$\epsilon_{1}$ & + & $0.9164 \mathrm{e}-4$ & $\epsilon_{4}$ & + & 0.0001851 \\
$\epsilon_{2}$ & + & 0.0315 & $\epsilon_{3}$ & + & 0.0280 \\
$\theta_{1}$ & + & 0.4343 & $\theta_{1}$ & + & 0.4387 \\
$\theta_{2}$ & + & 0.4343 & $\theta_{2}$ & + & 0.4387 \\
\hline
\end{tabular}

The sensitivity indices are interpreted with regard to their magnitudes and signs. The basic reproduction number $\mathcal{R}_{0}$ increases (decreases) with the increase (decrease) in those parameters that have positive sensitivity indices. Conversely, it increases (decreases) with the decrease (increase) in those parameters that have negative sensitivity indices. Moreover, the magnitude of sensitivity indices represents the relative 
importance of those parameters that drive the transmission mechanism of the disease.

Clearly, when $\mathcal{R}_{0}=\mathcal{R}_{1}$ the most efficacious parameters is the biting rate of rural mosquitoes on humans $\beta_{1}$, which has a strong positive impact on the value of $\mathcal{R}_{0}$. While the most efficacious parameter when $\mathcal{R}_{0}=\mathcal{R}_{2}$ is the biting rate of the forest mosquitoes on primates $\beta_{3}$. Furthermore, the results highlight that the transmission probabilities $\theta_{1}, \quad{ }^{213}$ $\theta_{2}$ are as important as the ratios of population size $\alpha_{1}, \alpha_{3}$, in the disease transmission dynamics. Obviously, increasing the transmission probabilities will lead to an increase in $\mathcal{R}_{0}$. There are many factors affecting the transmission probabilities such as the ZIKV dose in the blood-meal as shown in 22], where authors have demonstrated that increasing ZIKV dose in the blood-meal significantly increases the probability of mosquitoes becoming infected, and consequently disseminating virus and becoming infectious. On the other hand, the recovery rate of humans $\gamma_{1}$ and the recovery rate of primates $\gamma_{2}$ have the most negative sensitive indices. Also, the short life span of mosquitoes will reduce the spread of the disease and hence decrease in $\mathcal{R}_{0}$. Thus, for controlling Zika virus, it is suggested to control the mosquito population and prevent their breeding near houses in rural area.

\section{Local Stability of the DFE}

Here, we discuss the local stability of the disease free equilibrium $\mathcal{Z}^{0}$ by finding the eigenvalues of the linearized system. The result is given in the following theorem:

Theorem 2 The DFE, given by $\mathcal{Z}^{0}$, of the model (2) is locally asymptotically stable if $R_{0}<1$. Otherwise, it is unstable.

proof

First, let us introduce the following:

$$
\delta_{1}=\lambda_{1}-\left[\gamma_{1}+\mu_{H}\left(1-\epsilon_{1}\right)\right], \quad \delta_{2}=\lambda_{2}-\left[\gamma_{2}+\mu_{P}\left(1-\epsilon_{4}\right)\right]
$$

Then, the linearized matrix of the system (2) at the disease free equilibrium $\mathcal{Z}^{0}$ is

$$
J_{\mathcal{Z}^{0}}=
$$




$\left[\begin{array}{cccccccccc}-\mu_{H} & -\left(\mu_{H} \epsilon_{1}+\lambda_{1}\right) & 0 & 0 & -\alpha_{1} \beta_{1} \theta_{1} & 0 & -\kappa \alpha_{2} \beta_{2} \theta_{1} & 0 & 0 & 0 \\ 0 & \delta_{1} & 0 & 0 & \alpha_{1} \beta_{1} \theta_{1} & 0 & \kappa \alpha_{2} \beta_{2} \theta_{1} & 0 & 0 & 0 \\ 0 & \gamma_{1} & -\mu_{H} & 0 & 0 & 0 & 0 & 0 & 0 & 0 \\ 0 & -\beta_{1} \theta_{2} & 0 & -\mu_{V} & -\mu_{V} \epsilon_{2} & 0 & 0 & 0 & 0 & 0 \\ 0 & \beta_{1} \theta_{2} & 0 & 0 & -\mu_{V}\left(1-\epsilon_{2}\right) & 0 & 0 & 0 & 0 & 0 \\ 0 & 0 & 0 & 0 & 0 & -\mu_{U} & -\mu_{U} \epsilon_{3} & 0 & -\beta_{3} \theta_{2} & 0 \\ 0 & 0 & 0 & 0 & 0 & 0 & -\mu_{U}\left(1-\epsilon_{3}\right) & 0 & \beta_{3} \theta_{2} & 0 \\ 0 & 0 & 0 & 0 & 0 & 0 & -\alpha_{3} \beta_{3} \theta_{1} & -\mu_{P} & -\left(\mu_{P} \epsilon_{4}+\lambda_{2}\right) & 0 \\ 0 & 0 & 0 & 0 & 0 & 0 & \alpha_{3} \beta_{3} \theta_{1} & 0 & \delta_{2} & 0 \\ 0 & 0 & 0 & 0 & 0 & 0 & 0 & 0 & \gamma_{2} & -\mu_{P}\end{array}\right]$.

Clearly, the above system has six negative eigenvalues which are:

$$
-\mu_{H},-\mu_{H},-\mu_{V},-\mu_{U},-\mu_{P},-\mu_{P}
$$

The remaining eigenvalues satisfy the characteristic equation $P(\lambda)=0$, which is given by

$$
P(\lambda):=\lambda^{4}+p_{1} \lambda^{3}+p_{2} \lambda^{2}+p_{3} \lambda+p_{4}
$$


where

$$
\begin{aligned}
p_{1} & =\mu_{U}\left(1-\epsilon_{3}\right)+\mu_{V}\left(1-\epsilon_{2}\right)+\left[\mu_{H}\left(1-\epsilon_{1}\right)+\gamma_{1}\right]\left(1-\mathcal{R}_{H H}\right)+\left[\mu_{P}\left(1-\epsilon_{4}\right)+\gamma_{2}\right] \\
& \times\left(1-\mathcal{R}_{P P}\right), \\
p_{2} \quad & =\mu_{U}\left(1-\epsilon_{3}\right)\left[\gamma_{2}+\mu_{P}\left(1-\epsilon_{4}\right)\right]\left[1-\left(\mathcal{R}_{P P}+\mathcal{R}_{P U}\right)\right]+\mu_{V}\left(1-\epsilon_{2}\right)\left[\mu_{H}\left(1-\epsilon_{1}\right)+\gamma_{1}\right] \\
& {\left[1-\left(\mathcal{R}_{H H}+\mathcal{R}_{H V}\right)\right]+\left[\mu_{U}\left(1-\epsilon_{3}\right)+\left[\mu_{P}\left(1-\epsilon_{4}\right)+\gamma_{2}\right]\left(1-\mathcal{R}_{P P}\right)\right]+\left[\mu_{V}\left(1-\epsilon_{2}\right)\right.} \\
& \left.+\left[\mu_{H}\left(1-\epsilon_{1}\right)+\gamma_{1}\right]\left(1-\mathcal{R}_{H H}\right)\right], \\
& =\left[\mu_{V}\left(1-\epsilon_{2}\right)+\left[\mu_{H}\left(1-\epsilon_{1}\right)+\gamma_{1}\right]\left(1-\mathcal{R}_{H H}\right)\right] \mu_{U}\left(1-\epsilon_{3}\right)\left[\mu_{H}\left(1-\epsilon_{1}\right)+\gamma_{1}\right] \\
& \times\left[1-\left(\mathcal{R}_{P P}+\mathcal{R}_{P U}\right)\right] \\
& +\left[\mu_{U}\left(1-\epsilon_{3}\right)+\left[\mu_{P}\left(1-\epsilon_{4}\right)+\gamma_{2}\right]\left(1-\mathcal{R}_{P P}\right)\right] \mu_{V}\left(1-\epsilon_{2}\right)\left[\mu_{H}\left(1-\epsilon_{1}\right)+\gamma_{1}\right] \\
& \times\left[1-\left(\mathcal{R}_{H H}+\mathcal{R}_{H V}\right)\right], \\
& \times\left[1-\left(\mathcal{R}_{P P}+\mathcal{R}_{P U}\right)\right] . \\
p_{3} & =\mu_{V} \mu_{U}\left(1-\epsilon_{2}\right)\left(1-\epsilon_{3}\right)\left[\mu_{H}\left(1-\epsilon_{1}\right)+\gamma_{1}\right]\left[\mu_{P}\left(1-\epsilon_{4}\right)+\gamma_{2}\right]\left[1-\left(\mathcal{R}_{H H}+\mathcal{R}_{H V}\right)\right] \\
&
\end{aligned}
$$

Obviously, $p_{4}>0$ when $\mathcal{R}_{H H}+\mathcal{R}_{H V}<1$ and $\mathcal{R}_{P P}+\mathcal{R}_{P U}<1$ which implies that $p_{1}>0, p_{2}>0$ and $p_{3}>0$. Hence, the necessary conditions of stability using Routh's stability criterion [23] are satisfied. It is left to show that the sufficient conditions, namely, $p_{1} p_{2}-p_{3}>0$ and $p_{1} p_{2} p_{3}-p_{3}^{2}-p_{1}^{2} p_{4}>0$ are satisfied. This can be done by computing

$$
p_{1} p_{2}-p_{3}=
$$$$
\left[\mu_{V}\left(1-\epsilon_{2}\right)+\left[\mu_{H}\left(1-\epsilon_{1}\right)+\gamma_{1}\right]\left(1-\mathcal{R}_{H H}\right)\right] \mu_{V}\left(1-\epsilon_{2}\right)\left[\mu_{H}\left(1-\epsilon_{1}\right)+\gamma_{1}\right]
$$$$
\times\left[1-\left(\mathcal{R}_{H H}+\mathcal{R}_{H V}\right)\right]+\left[\mu_{U}\left(1-\epsilon_{3}\right)+\left[\mu_{P}\left(1-\epsilon_{4}\right)+\gamma_{2}\right]\left(1-\mathcal{R}_{P P}\right)\right]
$$$$
\times \mu_{U}\left(1-\epsilon_{3}\right)\left[\mu_{P}\left(1-\epsilon_{4}\right)+\gamma_{2}\right]\left[1-\left(\mathcal{R}_{P P}+\mathcal{R}_{P U}\right)\right]+\left[\mu_{V}\left(1-\epsilon_{2}\right)+\right.
$$$$
\left.\left[\mu_{H}\left(1-\epsilon_{1}\right)+\gamma_{1}\right]\left(1-\mathcal{R}_{H H}\right)\right]\left[\mu_{U}\left(1-\epsilon_{3}\right)+\left[\mu_{P}\left(1-\epsilon_{4}\right)+\gamma_{2}\right]\left(1-\mathcal{R}_{P P}\right)\right]
$$

and

$$
p_{1} p_{2} p_{3}-p_{3}^{2}-p_{1}^{2} p_{4}=
$$




$$
\begin{aligned}
& {\left[\mu_{V}\left(1-\epsilon_{2}\right)+\left[\mu_{H}\left(1-\epsilon_{1}\right)+\gamma_{1}\right]\left(1-\mathcal{R}_{H H}\right)\right]^{2}\left[\mu_{U}\left(1-\epsilon_{3}\right)+\left[\mu_{P}\left(1-\epsilon_{4}\right)+\gamma_{2}\right]\left(1-\mathcal{R}_{P P}\right)\right]^{2}} \\
& \left\{\mu_{V}\left(1-\epsilon_{2}\right)\left[\mu_{H}\left(1-\epsilon_{1}\right)+\gamma_{1}\right]\left[1-\left(\mathcal{R}_{H H}+\mathcal{R}_{H V}\right)\right]+\mu_{U}\left(1-\epsilon_{3}\right)\left[\mu_{P}\left(1-\epsilon_{4}\right)+\gamma_{2}\right]\right. \\
& \left.\times\left[1-\left(\mathcal{R}_{P P}+\mathcal{R}_{P U}\right)\right]\right\}+\left[\mu_{V}\left(1-\epsilon_{2}\right)+\left[\mu_{H}\left(1-\epsilon_{1}\right)+\gamma_{1}\right]\left(1-\mathcal{R}_{H H}\right)\right]\left[\mu_{U}\left(1-\epsilon_{3}\right)+\right. \\
& \left.\left[\mu_{P}\left(1-\epsilon_{4}\right)+\gamma_{2}\right]\left(1-\mathcal{R}_{P P}\right)\right] \times\left\{\left[\mu_{V}\left(1-\epsilon_{2}\right)\left[\mu_{H}\left(1-\epsilon_{1}\right)+\gamma_{1}\right]\left[1-\left(\mathcal{R}_{H H}+\mathcal{R}_{H V}\right)\right]\right.\right. \\
& \left.+\mu_{U}\left(1-\epsilon_{3}\right)\left[\mu_{P}\left(1-\epsilon_{4}\right)+\gamma_{2}\right]\left[1-\left(\mathcal{R}_{P P}+\mathcal{R}_{P U}\right)\right]\right]^{2}+\left[\mu_{U}\left(1-\epsilon_{3}\right)+\left[\mu_{P}\left(1-\epsilon_{4}\right)+\gamma_{2}\right]\right. \\
& \left.\times\left(1-\mathcal{R}_{P P}\right)\right]^{2} \mu_{V}\left(1-\epsilon_{2}\right)\left[\mu_{H}\left(1-\epsilon_{1}\right)+\gamma_{1}\right]\left[1-\left(\mathcal{R}_{H H}+\mathcal{R}_{H V}\right)\right]+\left[\mu_{V}\left(1-\epsilon_{2}\right)+\right. \\
& \left.\left.\left[\mu_{H}\left(1-\epsilon_{1}\right)+\gamma_{1}\right]\left(1-\mathcal{R}_{H H}\right)\right]^{2} \mu_{U}\left(1-\epsilon_{3}\right)\left[\mu_{P}\left(1-\epsilon_{4}\right)+\gamma_{2}\right]\left[1-\left(\mathcal{R}_{P P}+\mathcal{R}_{P U}\right)\right]\right\} . \\
& \text { Clearly, } p_{1} p_{2}-p_{3}>0 \text { and } p_{1} p_{2} p_{3}-p_{3}^{2}-p_{1}^{2} p_{4}>0 \text { if and only if }
\end{aligned}
$$

$$
\mathcal{R}_{H H}+\mathcal{R}_{H V}<1 \text { and } \mathcal{R}_{P P}+\mathcal{R}_{P U}<1
$$

Hence, the eigenvalues of characteristic equation $p(\lambda)=0$ have negative real parts whenever $R_{0}<1$, which implies that the $\operatorname{DFE}\left(\mathcal{Z}^{0}\right)$ is locally asymptotically stable.

\section{Global Stability of the DFE}

The global stability of the DFE $\left(\mathcal{Z}^{0}\right)$ will ensure that the disease is eliminated under all initial conditions. In this regard, we state the following theorem:

Theorem 3 The disease free equilibrium, given by $\mathcal{Z}^{0}$ of the model (2) is globally asymptotically stable if $R_{0}<1$.

proof

The proof is based on using Castillo-Chavez theorem [24]. Let $X(t)$ and $Y(t)$ compartments describe the uninfected and infected classes of system (2), respectively:

$$
X(t)=\left[\begin{array}{c}
S_{H}(t) \\
R_{H}(t) \\
S_{V}(t) \\
S_{U}(t) \\
S_{P}(t) \\
R_{P}(t)
\end{array}\right], \quad Y(t)=\left[\begin{array}{c}
I_{H}(t) \\
I_{V}(t) \\
I_{U}(t) \\
I_{P}(t)
\end{array}\right] .
$$

Therefore, system (2) can be written as : 


$$
\frac{d X}{d t}=F(X, Y), \quad \frac{d Y}{d t}=G(X, Y) ; \quad G(X, 0)=0
$$

where $F$ and $G$ are the corresponding right hand sides in system (2).

According to Castillo-Chavez theorem, in order to guarantee the global asymptotic stability of the $\operatorname{DFE}\left(\mathcal{Z}^{0}\right)$, the following two conditions $(H 1)$ and $(H 2)$ must be satisfied:

- $(H 1) X^{0}=(1,0,1,1,1,0)^{T}$ is globally asymptotically stable for $\frac{d X}{d t}=F(X, 0)$.

- $(H 2) \hat{G} \geqslant 0$, where $\hat{G}(X, Y)=A Y-G(X, Y)$ and $A=D_{Y} G\left(X^{0}, 0\right)$ is an Metzler matrix $\forall(X, Y) \in \Omega$.

First to check $(H 1)$, we have

$$
\frac{d X}{d t}=F(X, 0)=\left[\begin{array}{c}
-\mu_{H} S_{H}+\mu_{H} \\
-\mu_{H} R_{H} \\
-\mu_{V} S_{V}+\mu_{V} \\
-\mu_{U} S_{U}+\mu_{U} \\
-\mu_{P} S_{P}+\mu_{P} \\
-\mu_{P} R_{P}
\end{array}\right]
$$

then, the behavior of each compartment can be obtained by solving the above system of ODEs and hence, we get

$$
\left[\begin{array}{c}
S_{H} \\
R_{H} \\
S_{V} \\
S_{U} \\
S_{P} \\
R_{P}
\end{array}\right]=\left[\begin{array}{c}
1+S_{H}(0) e^{-\mu_{H} t} \\
R_{H}(0) e^{-\mu_{H} t} \\
1+S_{V}(0) e^{-\mu_{V} t} \\
1+S_{U}(0) e^{-\mu_{U} t} \\
1+S_{P}(0) e^{-\mu_{P} t} \\
R_{P}(0) e^{-\mu_{P} t}
\end{array}\right]
$$

Clearly, $\lim _{t \rightarrow \infty} X(t)=X^{0}$, i.e, the first condition is satisfied. Next, we check the second condition (H2) by finding $A=D_{Y} G\left(X^{0}, 0\right)$ : 
$A=\left[\begin{array}{cccc}-\mu_{H}\left(1-\epsilon_{1}\right)+\lambda_{1}-\gamma_{1} & \beta_{1} \theta_{1} \alpha_{1} & \kappa \beta_{2} \theta_{1} \alpha_{2} & 0 \\ \beta_{1} \theta_{2} & -\mu_{V}\left(1-\epsilon_{2}\right) & 0 & 0 \\ 0 & 0 & -\mu_{U}\left(1-\epsilon_{3}\right) & \beta_{3} \theta_{2} \\ 0 & 0 & \alpha_{3} \beta_{3} \theta_{1} & -\mu_{p}\left(1-\epsilon_{4}\right)+\lambda_{2}-\gamma_{1}\end{array}\right]$,

then, we compute $\hat{G}(X, Y)=A Y-G(X, Y)$ :

$$
\hat{G}=\left[\begin{array}{c}
\left(\alpha_{1} \beta_{1} \theta_{1} I_{V}+\kappa \alpha_{2} \beta_{2} \theta_{1} I_{U}+\lambda_{1} I_{H}\right)\left(1-S_{H}\right) \\
\beta_{1} \theta_{2} I_{H}\left(1-S_{V}\right) \\
\beta_{3} \theta_{2} I_{P}\left(1-S_{U}\right) \\
\left(\alpha_{3} \beta_{3} \theta_{1} I_{U}+\lambda_{2} I_{P}\right)\left(1-S_{P}\right)
\end{array}\right]
$$

Clearly, $\hat{G} \geqslant 0, \forall(X, Y) \in \Omega$ since $0 \leqslant\left(S_{H}, S_{V}, S_{U}, S_{P}\right) \leqslant 1$. Thus, (H2) is satisfied. $\quad{ }_{274}^{274}$

Hence, $\mathcal{Z}^{0}$ is globally asymptotically stable, provided that $\mathcal{R}_{0}<1$.

\section{Existence of Endemic Equilibria}

In this section, result related to the existence of axial and endemic equilibria is stated in $\quad 277$ the following theorem:

Theorem 4 The model (2) poses two endemic equilibrium points:

(i) a unique Axial equilibrium (AE) given by $\mathcal{Z}^{(1)}$, exists if $\mathcal{R}_{1}>1$,

(ii) a full Endemic equilibrium(EE) given by $\mathcal{Z}^{(2)}$, exists if $\mathcal{R}_{2}>1$.

proof

Let $\mathcal{Z}^{*}:=\left(S_{H}^{*}, I_{H}^{*}, R_{H}^{*}, S_{V}^{*}, I_{V}^{*}, S_{U}^{*}, I_{U}^{*}\right)$ be the expected endemic equilibrium of the model (2). By solving 


$$
\begin{aligned}
& S_{H}^{\prime}=0, R_{H}^{\prime}=0, S_{V}^{\prime}=0, I_{V}^{\prime}=0, S_{U}^{\prime}=0, I_{V}^{\prime}=0, S_{P}^{\prime}=0, R_{P}^{\prime}=0, \text { we get } \\
& S_{H}^{*}=\frac{\mu_{H}-\left(\mu_{H}+\gamma_{1}\right) I_{H}^{*}}{\mu_{H}}, \quad R_{H}^{*}=\frac{\gamma_{1} I_{H}^{*}}{\mu_{H}}, \quad S_{V}^{*}=\frac{\mu_{V}\left(1-\epsilon_{2}\right)}{\mu_{V}\left(1-\epsilon_{2}\right)+\beta_{1} \theta_{2} I_{H}^{*}}, \\
& I_{V}^{*}=\frac{\beta_{1} \theta_{2} I_{H}^{*}}{\mu_{V}\left(1-\epsilon_{2}\right)+\beta_{1} \theta_{2} I_{H}^{*}}, \quad S_{U}^{*}=\frac{\mu_{U}\left(1-\epsilon_{3}\right)}{\mu_{U}\left(1-\epsilon_{3}\right)+\beta_{3} \theta_{2} I_{P}^{*}}, \quad I_{U}^{*}=\frac{\beta_{3} \theta_{2} I_{P}^{*}}{\mu_{U}\left(1-\epsilon_{3}\right)+\beta_{3} \theta_{2} I_{P}^{*}} \\
& S_{P}^{*}=\frac{\mu_{P}-\left(\mu_{P}+\gamma_{2}\right) I_{P}^{*}}{\mu_{P}}, \quad R_{P}^{*}=\frac{\gamma_{2} I_{P}^{*}}{\mu_{P}},
\end{aligned}
$$

then by solving $I_{H}^{\prime}=0$ and using the expression of $S_{H}^{*}$, we get:

$$
\beta_{1} \alpha_{1} \theta_{1} I_{V}^{*}+\kappa \beta_{2} \theta_{1} \alpha_{2} I_{U}^{*}=\frac{\mu_{H}\left[\gamma_{1}+\mu_{H}\left(1-\epsilon_{1}\right)\right] I_{H}^{*}-\lambda_{1}\left[\mu_{H}-\left(\mu_{H}+\gamma_{1}\right) I_{H}^{*}\right] I_{H}^{*}}{\mu_{H}-\left(\mu_{H}+\gamma_{1}\right) I_{H}^{*}}
$$

Substitution of $I_{V}^{*}$ and $I_{U}^{*}$ into equation (9) leads to the following equation in terms of $I_{H}^{*}$ and $I_{P}^{*}$ :

$$
\frac{\beta_{3} \theta_{2} I_{P}^{*}}{\mu_{U}\left(1-\epsilon_{3}\right)+\beta_{3} \theta_{2} I_{P}^{*}}=\frac{a_{1} I_{H}^{* 3}+a_{2} I_{H}^{* 2}+a_{3} I_{H}^{*}}{b_{1} I_{H}^{* 2}+b_{2} I_{H}^{*}+b_{3}}
$$

where

$$
\begin{aligned}
a_{1} & =\beta_{1} \lambda_{1} \theta_{2}\left(\mu_{H}+\gamma_{1}\right), \\
a_{2} & =\mu_{V}\left(\mu_{H}+\gamma_{1}\right)\left(1-\epsilon_{2}\right)\left(\gamma_{1}+\mu_{H}\left(1-\epsilon_{1}\right)\right)\left[\mathcal{R}_{H H}+\mathcal{R}_{H V}\right] \\
& +\mu_{H} \beta_{1} \theta_{2}\left(\gamma_{1}+\mu_{H}\left(1-\epsilon_{1}\right)\right)\left[1-\mathcal{R}_{H H}\right], \\
a_{3} & =\mu_{V} \mu_{H}\left(1-\epsilon_{2}\right)\left(\gamma_{1}+\mu_{H}\left(1-\epsilon_{1}\right)\right)\left[1-\left(\mathcal{R}_{H H}+\mathcal{R}_{H V}\right)\right], \\
b_{1} & =-\beta_{1} \beta_{2} \theta_{2} \theta_{1} \alpha_{2}\left(\mu_{H}+\gamma_{1}\right), \\
b_{2} & =\beta_{2} \theta_{1} \alpha_{2}\left[\beta_{1} \theta_{2} \mu_{H}-\mu_{V}\left(1-\epsilon_{2}\right)\left(\mu_{H}+\gamma_{1}\right)\right], \\
b_{3} & =\beta_{2} \theta_{1} \alpha_{2} \mu_{V} \mu_{H}\left(1-\epsilon_{2}\right) .
\end{aligned}
$$

Solving $I_{P}^{\prime}=0$ and using expressions of $S_{P}^{*}$ and $I_{U}^{*}$ gives the following cubic equation:

$$
c_{1} I_{P}^{* 3}+c_{2} I_{P}^{* 2}+c_{3} I_{P}^{*}=0,
$$


where

$$
\begin{aligned}
c_{1} & =\beta_{3} \lambda_{2} \theta_{2} \mu_{H}\left(\gamma_{2}+\mu_{P}\right), \\
c_{2} & =\beta_{3}^{2} \theta_{1} \theta_{2} \alpha_{3} \mu_{H}\left[\gamma_{2}+\mu_{P}\right)+\lambda_{2} \mu_{U}\left(1-\epsilon_{3}\right)\left(\gamma_{2}+\mu_{P}\right)+\beta_{3} \theta_{2} \mu_{P}\left[\gamma_{2}+\mu_{P}\left(1-\epsilon_{4}\right)\right] \\
& {\left[1-\mathcal{R}_{P P}\right] } \\
c_{3} & =\mu_{P} \mu_{U}\left(1-\epsilon_{3}\right)\left[\gamma_{2}+\mu_{P}\left(1-\epsilon_{4}\right)\right]\left[1-\left(\mathcal{R}_{P P}+\mathcal{R}_{P U}\right)\right] .
\end{aligned}
$$

Now, from equation [11, we have the following two cases:

(i) if $I_{P}^{*}=0$, then equation 10 becomes a cubic equation in terms of $I_{H}^{*}$ :

$$
a_{1} I_{H}^{* 3}+a_{2} I_{H}^{* 2}+a_{3} I_{H}^{*}=0
$$

From the above equation, we have either $I_{H}^{*}=0$ which gives the DFE or we get the quadratic equation:

$$
a_{1} I_{H}^{* 2}+a_{2} I_{H}^{*}+a_{3}=0 .
$$

Clearly, $a_{1}>0$ and $a_{3}<0$ if and only if $\mathcal{R}_{H H}+\mathcal{R}_{H V}>1$, which implies $\mathcal{R}_{1}>1$. Thus, according to the Descartes's Sign Rule [25], equation [13 has a unique positive root. Moreover, since $I_{P}^{*}=0$, we have

$$
S_{U}^{*}=1, \quad I_{U}^{*}=0, \quad S_{P}^{*}=1, \quad R_{P}^{*}=0 .
$$

Hence, we have a unique $\mathrm{AE}$, which is given by

$$
\mathcal{Z}^{(1)}=\left(S_{H}^{*}, I_{H}^{*}, R_{H}^{*}, S_{V}^{*}, I_{V}^{*}, 1,0,1,0,0\right)
$$

where, $I_{H}^{*}$ satisfies equation 13 .

(ii) If $I_{P} \neq 0$, then solving equation (11) yields

$$
c_{1} I_{P}^{* 2}+c_{2} I_{P}^{*}+c_{3}=0
$$

which has a unique positive root $I_{P}^{*}$ if $\mathcal{R}_{2}>1$, since $c_{1}>0$ and $c_{3}<0$ if and only if $\mathcal{R}_{P P}+\mathcal{R}_{P U}>1$.

Now, using the above result that $I_{P}^{*}$ is the unique positive root of 14 , equation 10 
can be written terms of $I_{H}^{*}$ only:

$$
d_{1} I_{H}^{* 3}+d_{2} I_{H}^{* 2}+d_{3} I_{H}^{*}+d_{4}=0
$$

such that

$$
\begin{aligned}
d_{1} & =a_{1}\left[\mu_{U}\left(1-\epsilon_{3}\right)+\kappa \beta_{2} \beta_{3} \alpha_{2} \theta_{1} \theta_{2}^{2} I_{P}^{*}\right], \\
d_{2} & =\left[\mu_{U}\left(1-\epsilon_{3}\right)-\kappa \beta_{2} \beta_{3}^{2} \alpha_{2} \theta_{1} \theta_{2}^{2} I_{P}^{*}\right]\left[\gamma_{1}+\mu_{H}\left(1-\epsilon_{1}\right)\right]\left[\mu_{V}\left(\gamma_{1}+\mu_{H}\right)\left(\mathcal{R}_{H H}+\mathcal{R}_{H V}\right)\right. \\
& \left.+\beta_{1} \theta_{2} \mu_{H}\left(1-\mathcal{R}_{H H}\right)\right]+\beta_{2} \beta_{3} \theta_{1} \theta_{2}^{2} \alpha_{2} I_{P}^{*}, \\
d_{3} & =\mu_{V} \mu_{H} \mu_{U}\left(1-\epsilon_{2}\right)\left(1-\epsilon_{3}\right)\left(1-\beta_{2} \beta_{3}^{2} \theta_{2} I_{P}^{*} \mathcal{R}_{H V}\right)\left[\gamma_{1}+\mu_{H}\left(1-\epsilon_{1}\right)\right]^{2} \\
& {\left[1-\left(\mathcal{R}_{H H}+\mathcal{R}_{H V}\right)\right]+\kappa \beta_{2} \beta_{3} \alpha_{2} \theta_{1} \theta_{2} I_{P}^{*} \mu_{V}\left(1-\epsilon_{2}\right)\left(\mu_{H}+\gamma_{1}\right), } \\
d_{4}= & -\kappa \beta_{2} \beta_{3} \alpha_{2} \theta_{1} \theta_{2} \mu_{H} \mu_{V}\left(1-\epsilon_{2}\right) I_{P}^{*} .
\end{aligned}
$$

Hence, applying Descartes's Sign Rule, provided that $I_{P}^{*}$ is positive if $\mathcal{R}_{2}>1, d_{1}>0 \quad{ }_{298}$ and $d_{4}<0$, we have the following possibilities for the existence of positive roots of $\quad 299$ equation (15):

(I) a unique positive root, if $d_{2}>0$ and $d_{3}<0$ or both $d_{2}$ and $d_{3}$ have the same sign.

(II) three positive roots, if $d_{2}<0$ and $d_{3}>0$.

Thus, EE exits if $\mathcal{R}_{2}>1$ and can be written as

$$
\mathcal{Z}^{(2)}=\left(S_{H}^{*}, I_{H}^{*}, R_{H}^{*}, S_{V}^{*}, I_{V}^{*}, S_{U}^{*}, I_{U}^{*}, S_{P}^{*}, I_{P}^{*}, R_{P}^{*}\right)
$$

where, $I_{P}^{*}$ and $I_{H}^{*}$ can be computed using equations (14) and (15), respectively.

\section{Bifurcation and Local Stability Analysis}

Emergence or disappearance of new stable points causing a changes in stability of a system as a parameter changes is defined as bifurcation. In this section, we discuss the bifurcation of system (2) numerically and theoretically. All figures in this section is sketched using Matcont program 26.

Here, we take the transmission probability from an infectious mosquito, $\theta_{1}$, as a 
bifurcation parameter for which there are two possible bifurcations values,

$$
\theta_{1}^{*}=\frac{\mu_{V}\left(1-\epsilon_{2}\right)\left[\gamma_{1}+\mu_{H}\left(1-\epsilon_{1}\right)\right]\left(1-\mathcal{R}_{H H}\right)}{\beta_{1}^{2} \theta_{2} \alpha_{1}}=\frac{\delta_{H}}{\beta_{1}^{2} \theta_{2} \alpha_{1}}, \quad \mathcal{R}_{H H}<1,
$$

and

$$
\breve{\theta}_{1}=\frac{\mu_{U}\left(1-\epsilon_{3}\right)\left[\gamma_{2}+\mu_{P}\left(1-\epsilon_{4}\right)\right]\left(1-\mathcal{R}_{P P}\right)}{\beta_{3}^{2} \theta_{2} \alpha_{3}}=\frac{\delta_{P}}{\beta_{3}^{2} \theta_{2} \alpha_{3}}, \quad \mathcal{R}_{P P}<1
$$

corresponding to $\mathcal{R}_{1}=1$ and $\mathcal{R}_{2}=1$, respectively, where

$\delta_{H}=\mu_{V}\left(1-\epsilon_{2}\right)\left[\gamma_{1}+\mu_{H}\left(1-\epsilon_{1}\right)\right]\left(1-\mathcal{R}_{H H}\right)$ and

$\delta_{P}=\mu_{U}\left(1-\epsilon_{3}\right)\left[\gamma_{2}+\mu_{P}\left(1-\epsilon_{4}\right)\right]\left(1-\mathcal{R}_{P P}\right)$.

Clearly, $\theta_{2}$ is the only common parameter between $\theta_{1}^{*}$ and $\breve{\theta}_{1}$. So, fixing the values of other parameters, $\theta_{1}^{*}$ and $\breve{\theta}_{1}$ will attain their maximum values of one when

$$
\theta_{2}=\frac{\delta_{H}}{\beta_{1}^{2} \alpha_{1}}=\theta_{2_{H}} \text { and } \theta_{2}=\frac{\delta_{P}}{\beta_{3}^{2} \alpha_{3}}=\theta_{2_{P}}
$$

respectively, and their minimum values are obtained when $\theta_{2}=1$. Hence, the feasibility of the bifurcation values $\theta_{1}^{*}$ and $\breve{\theta}_{1}$ depends on the value of $\theta_{2}$. In particular, if the value of $\theta_{2}$ is taking to be less than $\theta_{2_{H}}$ and $\theta_{2_{P}}$, then both bifurcation values will be greater than one and thus no bifurcation will take place. The bifurcation of the system (2) will now be examined numerically for the following two cases:

(a) $\mathcal{R}_{0}=\mathcal{R}_{1}>\mathcal{R}_{2}$ (b) $\mathcal{R}_{0}=\mathcal{R}_{2}>\mathcal{R}_{1}$ with $\theta_{1}$ as a bifurcation parameter, taking different values of $\theta_{2}$ and fixing the values of all other parameters. The bifurcation diagrams are illustrated in Figures $3-5$

Fig 3. Forward bifurcation at the DFE with $\theta_{2_{H}}<\theta_{2}=0.05<\theta_{2_{P}}$ for the case $\mathcal{R}_{0}=\mathcal{R}_{1}>\mathcal{R}_{2}$.

Fig 4. Double forward bifurcation at the DFE and AE, respectively, with $\theta_{2_{H}}<\theta_{2_{P}}<\theta_{2}=0.1$ for the case $\mathcal{R}_{0}=\mathcal{R}_{1}>\mathcal{R}_{2}$.

Fig 5. Forward bifurcation at the DFE with $\theta_{2_{P}}<\theta_{2_{H}}<\theta_{2}=0.07$ for the case $\mathcal{R}_{0}=\mathcal{R}_{2}>\mathcal{R}_{1}$.

In Figures 3 and 4 , we assume that $\beta_{1}=0.35, \beta_{2}=0.32, \beta_{3}=0.3, \lambda_{1}=0.1$, and 
$\theta_{2_{P}}=0.0578$. Choosing $\theta_{2}<\theta_{2_{H}}, \mathcal{R}_{0}$ will be less than unity for all values of $\theta_{1}$ and $\quad{ }_{325}$ hence the DFE is stable and no bifurcation will take place. Here, both $\theta_{1}^{*}$ and $\breve{\theta}_{1}$ are not 326 feasible. For example, when $\theta_{2}=0.01$, the critical values are $\theta_{1}^{*}=1.88$ and $\breve{\theta}_{1}=5.78 . \quad{ }^{327}$ The bifurcation diagram for the case $0.0188<\theta_{2}<0.0578$ is illustrated in Fig 3 . Here, 328 the transmission probability of infection to mosquitoes is taken to be $\theta_{2}=0.05$ and the ${ }^{329}$ corresponding critical values are $\theta_{1}^{*}=0.3761$ and $\breve{\theta}_{1}=1.1554$, which is not feasible. $\quad 330$ Clearly, the system undergoes forward bifurcation when $\theta_{1}$ passes through the critical 331 value $\theta_{1}^{*}$ as shown in the Figure. Furthermore, when $\theta_{2}$ is taken to be 0.1 , which is 332 greater than both $\theta_{2_{H}}$ and $\theta_{2_{P}}$, the critical values are $\theta_{1}^{*}=0.1880$ and $\breve{\theta}_{1}=0.5777$. In $\quad 333$ this case, the system has double forward bifurcation when $\theta_{1}$ passes through these two 334 critical values as shown in Fig 4 . The system undergoes forward bifurcation at the DFE 335 as $\theta_{1}$ passes through $\theta_{1}^{*}$ and at the $\mathrm{AE}$ as $\theta_{1}$ passes through $\breve{\theta}_{1}$.

For the case $R_{0}=\mathcal{R}_{2}>\mathcal{R}_{1}$, we choose $\beta_{1}=0.35, \beta_{2}=0.32, \beta_{3}=0.3, \lambda_{1}=0.0025$, and $\lambda_{2}=0.1$, the corresponding values of $\theta_{2_{P}}$ and $\theta_{2_{H}}$ are 0.0293 and 0.0450, respectively. Similar to the previous case, the feasibility of the critical values of $\theta_{1}$ depends on the value of the $\theta_{2}$ and its relation to $\theta_{2_{H}}$ and $\theta_{2_{P}}$. For illustration, we choose $\theta_{2}=0.07$ and the bifurcation diagram for this case is illustrated in Fig 5 . The figure shows that the system has forward bifurcation at the DFE when $\theta_{1}$ passes through $\breve{\theta}_{1}=0.4180$ and that the EE once exists, it remains stable. Moreover, unstable $\mathrm{AE}$ exists when the system passes through the second critical value $\theta_{1}^{*}=0.6427$ and hence there is no bifurcation at $\theta_{1}^{*}$.

Next, the bifurcation result will be summarized in the following theorem and it will confirmed using Sotomayor theorem as described in 27.

Theorem 5 The following holds:

(i) If $\mathcal{R}_{2}>\mathcal{R}_{1}$, then model (2) undergoes transcritical bifurcation at the DFE, given by $\mathcal{Z}^{0}$, when the parameter $\theta_{1}$ passes through the bifurcation value $\breve{\theta}_{1}$.

(ii) If $\mathcal{R}_{1}>\mathcal{R}_{2}$, then model (2) undergoes transcritical bifurcation at the DFE, given by $\mathcal{Z}^{0}$, when the parameter $\theta_{1}$ passes through the bifurcation value $\theta_{1}^{*}$ and at the ${ }_{352}$ $A E$, given by $\mathcal{Z}^{(1)}$, when the parameter $\theta_{1}$ passes through the bifurcation value $\breve{\theta}_{1} . \quad{ }_{353}$

proof We begin with the proof of part (i). Define the right hand side of system (2) 
as $Y(Z, t)$ such that

$$
Z=\left(S_{H}, I_{H}, R_{H}, S_{V}, I_{V}, S_{U}, I_{U}, S_{P}, I_{P}, R_{P}\right)^{T}
$$

At $\mathcal{R}_{0}=\mathcal{R}_{2}=1$ and $\mathcal{R}_{1}<1$, choose $\theta_{1}$ as a bifurcation parameter as define in 17 . ${ }^{354}$ The linearization matrix of system $(2)$ around the DFE, $\mathcal{Z}^{0}$ and when $\theta_{1}=\breve{\theta}_{1}$ is

$$
D_{Z} Y=
$$

$\left[\begin{array}{cccccccccc}-\mu_{H} & -\left(\mu_{H} \epsilon_{1}+\lambda_{1}\right) & 0 & 0 & \frac{-\alpha_{1} \beta_{1} \delta_{P}}{\beta_{3}^{2} \alpha_{3} \theta_{2}} & 0 & \frac{-\kappa \beta_{2} \alpha_{2} \delta_{P}}{\beta_{3}^{2} \alpha_{3} \theta_{2}} & 0 & 0 & 0 \\ 0 & \frac{-\delta_{H}}{\mu_{V}\left(1-\epsilon_{2}\right)} & 0 & 0 & \frac{\alpha_{1} \beta_{1} \delta_{P}}{\beta_{3}^{2} \alpha_{3} \theta_{2}} & 0 & \frac{\kappa \beta_{2} \alpha_{2} \delta_{P}}{\beta_{3}^{2} \alpha_{3} \theta_{2}} & 0 & 0 & 0 \\ 0 & \gamma_{1} & -\mu_{H} & 0 & 0 & 0 & 0 & 0 & 0 & 0 \\ 0 & -\beta_{1} \theta_{2} & 0 & -\mu_{V} & -\mu_{V} \epsilon_{2} & 0 & 0 & 0 & 0 & 0 \\ 0 & \beta_{1} \theta_{2} & 0 & 0 & -\mu_{V}\left(1-\epsilon_{2}\right) & 0 & 0 & 0 & 0 & 0 \\ 0 & 0 & 0 & 0 & 0 & -\mu_{U} & -\mu_{U} \epsilon_{3} & 0 & -\beta_{3} \theta_{2} & 0 \\ 0 & 0 & 0 & 0 & 0 & 0 & -\mu_{U}\left(1-\epsilon_{3}\right) & 0 & \beta_{3} \theta_{2} & 0 \\ 0 & 0 & 0 & 0 & 0 & 0 & \frac{-\delta_{P}}{\beta_{3} \theta_{2}} & -\mu_{P} & -\mu_{P} \epsilon_{4}-\lambda_{2} & 0 \\ 0 & 0 & 0 & 0 & 0 & 0 & \frac{\delta_{P}}{\beta_{3} \theta_{2}} & 0 & \frac{\delta_{P}}{\mu_{U}\left(1-\epsilon_{3}\right)} & 0 \\ 0 & 0 & 0 & 0 & 0 & 0 & 0 & 0 & \gamma_{2} & -\mu_{P}\end{array}\right]$.

One can easily check that the above matrix has a simple zero eigenvalue.

Then, the nonzero right eigenvector $v=\left(v_{1}, v_{2}, v_{3}, v_{4}, v_{5}, v_{6}, v_{7}, v_{8}, v_{9}, v_{10}\right)^{T}$ and the 358 nonzero left eigenvector $w=\left(w_{1}, w_{2}, w_{3}, w_{4}, w_{5}, w_{6}, w_{7}, w_{8}, w_{9}, w_{10}\right)^{T}$ associated with $\quad 359$ zero eigenvalue of $D_{Z} Y$ are

$$
v=\left[\begin{array}{c}
-\frac{\mu_{V}\left(\gamma_{1}+\mu_{H}\right)\left(1-\epsilon_{2}\right)}{\beta_{1} \theta_{2} \mu_{H}} v_{5} \\
\frac{\mu_{V}\left(1-\epsilon_{2}\right)}{\beta_{1} \theta_{2}} v_{5} \\
\frac{\gamma_{1} \mu_{V}\left(1-\epsilon_{2}\right)}{\beta_{1} \theta_{2} \mu_{H}} v_{5} \\
-v_{5} \\
v_{5} \\
-\frac{Q}{\kappa \beta_{1} \beta_{2} \alpha_{2} \delta_{P}} v_{5} \\
\frac{Q}{\kappa \beta_{1} \beta_{2} \alpha_{2} \delta_{P}} v_{5} \\
-\frac{\mu_{U}\left(1-\epsilon_{3}\right)\left(\gamma_{2}+\mu_{P}\right) Q}{\kappa \beta_{1} \beta_{2} \beta_{3} \theta_{2} \mu_{P} \delta_{P}} v_{5} \\
-\frac{\mu_{P}}{\left(\gamma_{2}+\mu_{p}\right) Q} v_{8} \\
-\frac{\gamma_{2}}{\gamma_{2}+\mu_{P}} v_{8}
\end{array}\right], \quad w=\left[\begin{array}{c}
0 \\
0 \\
0 \\
0 \\
\delta_{P} \\
\frac{\beta_{3} \theta_{2} \mu_{U}\left(1-\epsilon_{3}\right)}{w_{9}} \\
0 \\
w_{9} \\
0
\end{array}\right] \text {, }
$$


where $Q=\alpha_{3} \beta_{3}^{2} \delta_{H}-\alpha_{1} \beta_{1}^{2} \delta_{P}$. To check the first condition of Sotomayor theorem, we

$$
w^{T} Y_{\theta_{1}}\left(\breve{\theta}_{1}, \mathcal{Z}^{0}\right)=0
$$

and hence first condition is satisfied. Next, we compute the Jacobian of $Y_{\theta_{1}}(Z)$ at $\mathcal{Z}^{0}$ and $\theta_{1}=\breve{\theta}_{1}$ and we obtain

$$
D Y_{\theta_{1}}\left(\breve{\theta}_{1}, \mathcal{Z}^{0}\right)=\left[\begin{array}{cccccccccc}
0 & 0 & 0 & 0 & -\alpha_{1} \beta_{1} & 0 & -\kappa \alpha_{2} \beta_{2} & 0 & 0 & 0 \\
0 & 0 & 0 & 0 & \alpha_{1} \beta_{1} & 0 & \kappa \alpha_{2} \beta_{2} & 0 & 0 & 0 \\
0 & 0 & 0 & 0 & 0 & 0 & 0 & 0 & 0 & 0 \\
0 & 0 & 0 & 0 & 0 & 0 & 0 & 0 & 0 & 0 \\
0 & 0 & 0 & 0 & 0 & 0 & 0 & 0 & 0 & 0 \\
0 & 0 & 0 & 0 & 0 & 0 & 0 & 0 & 0 & 0 \\
0 & 0 & 0 & 0 & 0 & 0 & 0 & 0 & 0 & 0 \\
0 & 0 & 0 & 0 & 0 & 0 & -\alpha_{3} \beta_{3} & 0 & 0 & 0 \\
0 & 0 & 0 & 0 & 0 & 0 & \alpha_{3} \beta_{3} & 0 & 0 & 0 \\
0 & 0 & 0 & 0 & 0 & 0 & 0 & 0 & 0 & 0
\end{array}\right] .
$$

Then, we calculate

$$
w^{T} D Y_{\theta_{1}}\left(\breve{\theta}_{1}, \mathcal{Z}^{0}\right) v=\alpha_{3} \beta_{3} w_{9} v_{7} \neq 0
$$

therefore, the second condition is satisfied.

Since $D^{2}$ represents the matrix of partial derivatives of each components of $D Y$ with respect to each component of $Y(Z)$, we get 


$$
D^{2} Y\left(\breve{\theta}_{1}, \mathcal{Z}^{0}\right)(v, v)=\left[\begin{array}{c}
-3\left[\beta_{2} \kappa \alpha_{2} \theta_{1} v_{7}+\beta_{1} \theta_{1} \alpha_{1} v_{5}+\lambda_{1} v_{2}\right] v_{1} \\
3\left[\beta_{2} \kappa \alpha_{2} \theta_{1} v_{7}+\beta_{1} \theta_{1} \alpha_{1} v_{5}+\lambda_{1} v_{2}\right] v_{1} \\
0 \\
-4 \beta_{1} \theta_{2} v_{4} v_{2} \\
4 \beta_{1} \theta_{2} v_{2} v_{4} \\
-3 \beta_{3} \theta_{2} v_{6} v_{9} \\
3 \beta_{3} \theta_{2} v_{6} v_{9} \\
-\left[4 \beta_{3} \theta_{1} \alpha_{3} v_{7}+3 \lambda_{2} v_{9}\right] v_{8} \\
{\left[4 \beta_{3} \theta_{1} \alpha_{3} v_{7}+3 \lambda_{2} v_{9}\right] v_{8}} \\
0
\end{array}\right]
$$

Thus, the third condition is satisfied by showing that

$$
w^{T}\left[D^{2} Y\left(\breve{\theta}_{1}, \mathcal{Z}^{0}\right)(v, v)\right]=\frac{-2 Q^{2} w_{9} v_{5}^{2}}{\beta_{1}^{2} \beta_{2}^{2} \beta_{3} \alpha_{2}^{2} \theta_{2} \kappa^{2} \delta_{P}}\left[1+\frac{\mu_{U}\left(1-\epsilon_{3}\right)\left(\gamma_{2}+\mu_{P}\right)}{\beta_{3} \theta_{2} \mu_{P}}\right] \neq 0
$$

Hence, according to Sotomayor theorem, the results in $18,(19)$ and $(20)$ implies that as the parameter $\theta_{1}$ passes through the bifurcation value $\breve{\theta}_{1}$ defined in $[17$, the system 22 experiences a transcritical bifurcation at $\mathcal{Z}^{0}$ as shown on the figure above.

For the proof of part (ii), one can repeat the same procedure as we did for part (i). $\quad 374$ Choose $\theta_{1}^{*}$ as a bifurcation parameter defined in 16 . The left and right eigenvectors are 375

$$
w=\left[\begin{array}{c}
0 \\
-\frac{Q w_{9}}{\kappa \beta_{2} \beta_{3} \alpha_{2} \delta_{H}} \\
0 \\
0 \\
Q w_{9} \\
-\frac{\beta_{1}}{\kappa \beta_{1} \beta_{2} \beta_{3} \alpha_{2} \theta_{2} \mu_{V}\left(1-\epsilon_{2}\right)} \\
0 \\
\frac{\delta_{P} w_{9}}{\beta_{3} \theta_{2} \mu_{U}\left(1-\epsilon_{3}\right)} \\
0 \\
w_{9}
\end{array}\right], v\left[\begin{array}{c}
\frac{-\mu_{V}\left(1-\epsilon_{2}\right)\left(\gamma_{1}+\mu_{H}\right) v_{5}}{\beta_{1} \theta_{2} \mu_{H}} \\
\frac{\mu_{V}\left(1-\epsilon_{2}\right) v_{5}}{\beta_{1}} \\
-v_{5} \\
v_{5} \\
0 \\
0 \\
0 \\
0 \\
0
\end{array}\right] \text {, }
$$


respectively. The conditions of Sotomayor Theorem hold as follows:

$$
w^{T} D Y_{\theta_{1}}\left(\theta_{1}^{*}, \mathcal{Z}^{0}\right) v=\alpha_{1} \beta_{1} w_{2} v_{5} \neq 0
$$

and

$$
w^{T}\left[D^{2} Y\left(\theta_{1}^{*}, \mathcal{Z}^{0}\right)(v, v)\right]=\frac{2 Q w_{9} v_{5}^{2}}{\beta_{1} \beta_{2} \beta_{3} \kappa \alpha_{2} \theta_{2}}\left[1+\frac{\mu_{V}\left(1-\epsilon_{2}\right)\left(\gamma_{1}+\mu_{H}\right)}{\beta_{1} \theta_{2} \mu_{H}}\right] \neq 0
$$

Hence, the system (2) undergoes transcritical bifurcation at the DFE when the parameter $\theta_{1}$ passes through the bifurcation value $\theta_{1}=\theta_{1}^{*}$. Finally, proofing the bifurcation at the AE when $\theta_{1}$ passes through the bifurcation value $\theta_{1}=\breve{\theta}_{1}$ can be done in a similar way.

The local stability of endemic equilibria $\mathcal{Z}^{(1)}$ and $\mathcal{Z}^{(2)}$ can be established from the above calculations and Theorem 4 in 15 such that $a$ and $b$ are given by

$$
\begin{aligned}
& a=\frac{1}{2} w^{T}\left[D^{2} Y\left(\mathcal{Z}^{0}, \mu^{*}\right)(v, v)\right]=\frac{1}{2} \sum_{i, j, k=1}^{n} w_{i} v_{j} v_{k} \frac{\partial^{2} Y_{i}}{\partial x_{j} \partial x_{k}}\left(\mathcal{Z}^{0}, \mu^{*}\right), \\
& b=w^{T} D Y_{\mu}\left(\mathcal{Z}^{0}, \mu^{*}\right) v=\sum_{i, j=1}^{n} w_{i} v_{j} \frac{\partial^{2} Y_{i}}{\partial x_{j} \partial \mu}\left(\mathcal{Z}^{0}, \mu^{*}\right) .
\end{aligned}
$$

where $\mu^{*}$ is the bifurcation parameter and the result is given in the following theorem:

Theorem 6 The following results hold:

(i) Let $a$ and $b$ defined in (20) and (19) at $\theta_{1}=\breve{\theta}_{1}$ and $b \neq 0\left(w_{9}>0\right.$ and $\left.v_{5}>0\right)$. Then, there exists $\varepsilon_{1}>0$ such that the $A E$, given by $\mathcal{Z}^{(1)}$, is locally asymptotically stable near $\mathcal{Z}^{0}$ if $a<0$ for $0<\theta_{1}<\varepsilon_{1}$.

(ii) Let $a$ and $b$ defined in (22) and (21) at $\theta_{1}=\theta_{1}^{*}$ and $b \neq 0 \quad\left(w_{9}<0\right.$ and $\left.v_{5}>0\right)$.Then, there exists $\varepsilon_{2}>0$ such that the EE, given by $\mathcal{Z}^{(2)}$, is locally asymptotically stable near $\mathcal{Z}^{0}$ if $a<0$ for $0<\theta_{1}<\varepsilon_{2}$.

The bifurcation and stability results for the case $\mathcal{R}_{0}=\mathcal{R}_{1}>\mathcal{R}_{2}$ can be summarized as follows, taking $\theta_{1}$ as a bifurcation parameter and provided that the two critical values $\theta_{1}^{*}$ and $\breve{\theta}_{1}$ are feasible:

(i) When the transmission probability from an infectious mosquito is small $\theta_{1}<\theta_{1}^{*}<\breve{\theta}_{1}\left(\mathcal{R}_{2}<\mathcal{R}_{1}<1\right)$, then the number of infected will decrease and 
eventually the disease will disappear from both areas. This implies that the DFE is stable.

(ii) When $\theta_{1}=\theta_{1}^{*}<\breve{\theta}_{1}\left(\mathcal{R}_{2}<\mathcal{R}_{1}=1\right)$, the system undergoes forward bifurcation at the $\mathrm{DFE}$ as $\theta_{1}$ pass through bifurcation value $\theta_{1}^{*}$.

(iii) When $\theta_{1}^{*}<\theta_{1}<\breve{\theta}_{1}\left(\mathcal{R}_{2}<1<\mathcal{R}_{1}\right)$, the endemic will persists only in rural area and will die out from forest area. Hence, the AE is stable, while the DFE is unstable.

(iv) When $\theta_{1}^{*}<\theta_{1}=\breve{\theta}_{1}\left(1=\mathcal{R}_{2}<\mathcal{R}_{1}\right)$, the system undergoes forward bifurcation at the $\mathrm{AE}$ as $\theta_{1}$ pass through bifurcation value $\breve{\theta}_{1}$.

(v) When the transmission probability from an infectious mosquito is high $\theta_{1}^{*}<\breve{\theta}_{1}<\theta_{1}\left(\mathcal{R}_{1}>\mathcal{R}_{2}>1\right)$, the disease will persist in both rural and forest areas and hence the EE is stable, while both the DFE and AE are unstable.

Also, we summarize the bifurcation and stability results for the case $\mathcal{R}_{0}=\mathcal{R}_{2}>\mathcal{R}_{1}$ as follows:

(I) When $\theta_{1}<\breve{\theta}_{1}<\theta_{1}^{*}\left(\mathcal{R}_{1}<\mathcal{R}_{2}<1\right)$, then the disease will die out from both areas and hence the DFE is stable.

(II) When $\theta_{1}=\breve{\theta}_{1}<\theta_{1}^{*}\left(\mathcal{R}_{1}<\mathcal{R}_{2}=1\right)$, the system undergoes forward bifurcation at DFE as $\theta_{1}$ pass through bifurcation value $\breve{\theta}_{1}$.

(III) When $\breve{\theta}_{1}<\theta_{1}<\theta_{1}^{*}\left(\mathcal{R}_{1}<1<\mathcal{R}_{2}\right)$, the endemic will persist in both areas and hence the EE is stable, while the DFE is unstable.

(IV) When $\breve{\theta}_{1}<\theta_{1}=\theta_{1}^{*}\left(\mathcal{R}_{2}>\mathcal{R}_{1}=1\right)$, unstable AE exists as the system passes through $\theta_{1}^{*}$.

(V) When $\breve{\theta}_{1}<\theta_{1}^{*}<\theta_{1}\left(\mathcal{R}_{2}>\mathcal{R}_{1}>1\right)$, the EE remains stable and both the AE and DFE remain unstable.

Remark 1 One can choose $\theta_{2}$ as a bifurcation parameter with the following critical values:

$$
\theta_{2}^{*}=\frac{\delta_{H}}{\beta_{1}^{2} \theta_{1} \alpha_{1}}, \quad \mathcal{R}_{H H}<1
$$


which corresponds to $\mathcal{R}_{0}=\mathcal{R}_{1}=1, \mathcal{R}_{2}<1$ and

$$
\breve{\theta}_{2}=\frac{\delta_{P}}{\beta_{3}^{2} \theta_{1} \alpha_{3}}, \quad \mathcal{R}_{P P}<1
$$

which corresponds to $\mathcal{R}_{0}=\mathcal{R}_{2}=1, \mathcal{R}_{1}<1$ and get similar results with the feasibility of ${ }^{419}$ the two critical values depending on $\theta_{1}$.

From the above discussion, we note that the stability and bifurcation results depend on both transmission probability parameters $\theta_{1}$ and $\theta_{2}$. Hence, one may also study the combined effect of $\theta_{1}$ and $\theta_{2}$. So, let us introduce a new parameter $\Theta$ defined as $\Theta=\theta_{1} \theta_{2}$ with two critical values $\Theta^{*}=\frac{\delta_{H}}{\beta_{1}^{2} \alpha_{1}}$ and $\breve{\Theta}=\frac{\delta_{P}}{\beta_{3}^{2} \alpha_{3}}$. The stability region based on combined effect of $\theta_{1}$ and $\theta_{2}$ for the case $\mathcal{R}_{0}=\mathcal{R}_{1}>\mathcal{R}_{2}$ is shown in Fig 6 . From the figure, the stability and bifurcation results can be outlined as follows:

(i) When $\Theta<\Theta^{*}<\breve{\Theta}\left(\mathcal{R}_{2}<\mathcal{R}_{1}<1\right)$, the DFE is the only stable point. Thus, the disease will die out from both rural and forest areas.

(ii) The system undergoes a forward bifurcation at the DFE when $\Theta$ passes through critical value $\Theta^{*}$.

(iii) When $\Theta^{*}<\Theta<\breve{\Theta}\left(\mathcal{R}_{2}<1<\mathcal{R}_{1}\right)$, then the AE is the only stable point. Thus, the disease will persist in rural area and it will disappear from the forest area.

(iv) The system undergoes forward bifurcation at the $\mathrm{AE}$ when $\Theta$ passes through critical value $\breve{\Theta}$.

(v) When $\Theta^{*}<\breve{\Theta}<\Theta\left(\mathcal{R}_{1}>\mathcal{R}_{2}>1\right)$, the EE is the only stable point. Hence, the disease will persist in both rural and forest areas.

Fig 6. Stability region when $R_{0}=\mathcal{R}_{1}$

The stability region of combined effect of $\theta_{1}$ and $\theta_{2}$ for the case $\mathcal{R}_{0}=\mathcal{R}_{2}>\mathcal{R}_{1}$ is given in Fig 7 . The stability and bifurcation results based on combined effect $\Theta$ for this case can be outlined as follows:

(I) When $\Theta<\breve{\Theta}<\Theta^{*}\left(\mathcal{R}_{1}<\mathcal{R}_{2}<1\right)$, the DFE is the only stable point and the disease will die out from both areas. 
(II) The system undergoes forward bifurcation at the DFE when $\Theta$ passes through critical value $\breve{\Theta}$.

(III) When $\breve{\Theta}<\Theta<\Theta^{*}$ or $\breve{\Theta}<\Theta^{*}<\Theta$, the EE is the only stable point. In other words, if $\mathcal{R}_{2}>1$, the disease will persist in both areas.

Fig 7. Stability region when $R_{0}=\mathcal{R}_{2}$.

\section{Global Stability of Axial Equilibrium $\mathcal{Z}^{(1)}$}

In this section, we derive the global stability of the AE. The derivation is based on finding a suitable Lyapunov function and using the LaSalle's invariance principle. We begin by considering the Lyapunov function as follows:

$$
\mathcal{L}(t):=\mathcal{L}_{1}(t)+\mathcal{L}_{2}(t)
$$

such that

$$
\mathcal{L}_{1}(t):=\omega_{1} I_{U}(t)+\omega_{2} I_{P}(t)
$$

and

$$
\begin{aligned}
\mathcal{L}_{2}(t):= & \omega_{3}\left(S_{H}(t)-S_{H}^{*}-S_{H}^{*} \log \frac{S_{H}(t)}{S_{H}^{*}}\right)+\omega_{4}\left(I_{H}(t)-I_{H}^{*}-I_{H}^{*} \log \frac{I_{H}(t)}{I_{H}^{*}}\right) \\
& +\omega_{5}\left(S_{V}(t)-S_{V}^{*}-S_{V}^{*} \log \frac{S_{V}(t)}{S_{V}^{*}}\right)+\omega_{6}\left(I_{V}(t)-I_{V}^{*}-I_{V}^{*} \log \frac{I_{V}(t)}{I_{V}^{*}}\right) .
\end{aligned}
$$

Differentiating $\mathcal{L}(t)$ with respect to $t$ yields:

$$
\mathcal{L}^{\prime}(t)=\mathcal{L}_{1}^{\prime}(t)+\mathcal{L}_{2}^{\prime}(t)
$$

such that

$$
\mathcal{L}_{1}^{\prime}(t)=\omega_{1} I_{U}^{\prime}(t)+\omega_{2} I_{P}^{\prime}(t)
$$

and

$\mathcal{L}_{2}^{\prime}(t)=\omega_{3}\left(\frac{S_{H}-S_{H}^{*}}{S_{H}}\right) S_{H}^{\prime}+\omega_{4}\left(\frac{I_{H}-I_{H}^{*}}{I_{H}}\right) I_{H}^{\prime}+\omega_{5}\left(\frac{S_{V}-S_{V}^{*}}{S_{V}}\right) S_{V}^{\prime}+\omega_{6}\left(\frac{I_{V}-I_{V}^{*}}{I_{V}}\right) I_{V}^{\prime}$.

Substitution of $I_{U}^{\prime}$ and $I_{P}^{\prime}$ from system (2) gives 


$$
\begin{aligned}
\mathcal{L}_{1}^{\prime}(t) & =\omega_{1}\left[\mu_{U} \epsilon_{3} I_{U}+\beta_{3} \theta_{2} S_{U} I_{P}-\mu_{U} I_{U}\right]+ \\
\omega_{2}\left[\mu_{P} \epsilon_{4} I_{P}\right. & \left.+\beta_{3} \theta_{1} \alpha_{3} S_{P} I_{U}+\lambda_{2} S_{P} I_{P}-\left(\gamma_{2}+\mu_{P}\right) I_{P}\right], \\
\text { using } S_{U} & =1-I_{U} \text { and } S_{P}=1-I_{P}-R_{P} \text { yields } \\
L_{1}^{\prime}(t) & =\left[\omega_{1} \beta_{3} \theta_{2}+\omega_{2}\left(\lambda_{2}-\gamma_{2}-\mu_{P}\left(1-\epsilon_{4}\right)\right)\right] I_{P}+\left[\omega_{2} \beta_{3} \theta_{1} \alpha_{3}-\omega_{1} \mu_{U}\left(1-\epsilon_{3}\right)\right] I_{U} \\
& -\left[\omega_{1} \beta_{3} \theta_{2}+\omega_{2} \beta_{3} \theta_{1} \alpha_{3}\right] I_{P} I_{U}-\omega_{2} \lambda_{2} I_{P}^{2}-\omega_{2}\left[\lambda_{2} I_{P}+\beta_{3} \alpha_{3} \theta_{1} I_{U}\right] R_{P} .
\end{aligned}
$$

Next, choosing $\omega_{2}=1$ and $\omega_{1}=\frac{\beta_{3} \theta_{1} \alpha_{3}}{\mu_{U}\left(1-\epsilon_{3}\right)}$ gives

$$
\mathcal{L}_{1}^{\prime}(t)=-m+\left(\gamma_{2}+\mu_{P}\left(1-\epsilon_{4}\right)\right)\left[\left(\mathcal{R}_{P U}+\mathcal{R}_{P P}\right)-1\right] I_{P}
$$

such that $m=\left[\beta_{3} \theta_{1} \alpha_{3}\left(\frac{\beta_{3} \theta_{2}}{\mu_{U}\left(1-\epsilon_{3}\right)}+1\right) I_{P} I_{U}+\lambda_{2} I_{P}^{2}+\left(\lambda_{2} I_{P}+\beta_{3} \alpha_{3} \theta_{1} I_{U}\right) R_{P}\right]$.

Clearly, $\mathcal{L}_{1}^{\prime}(t)$ is negative definite when $\mathcal{R}_{2}<1$.

Moreover, substituting for $S_{H}^{\prime}, I_{H}^{\prime}, S_{V}^{\prime}$, and $I_{V}^{\prime}$ from system (2) into $\mathcal{L}_{2}^{\prime}(t)$ gives

$$
\begin{aligned}
& \mathcal{L}_{2}^{\prime}(t)=\omega_{3}\left(\frac{S_{H}-S_{H}^{*}}{S_{H}}\right)\left[\mu_{H}-\mu_{H} \epsilon_{1} I_{H}-\beta_{1} \theta_{1} \alpha_{1} I_{V} S_{H}-\kappa \beta_{2} \theta_{1} \alpha_{2} I_{U} S_{H}-\lambda_{1} I_{H} S_{H}\right. \\
& \left.\quad-\mu_{H} S_{H}\right]+\omega_{4}\left(\frac{I_{H}-I_{H}^{*}}{I_{H}}\right)\left[\mu_{H} \epsilon_{1} I_{H}+\left(\beta_{1} \theta_{1} \alpha_{1} I_{V} S_{H}+\kappa \beta_{2} \theta_{1} \alpha_{2} I_{U} S_{H}+\lambda_{1} I_{H} S_{H}\right)\right. \\
& \left.-\left(\gamma_{1}+\mu_{H}\right) I_{H}\right]+\omega_{5}\left(\frac{S_{V}-S_{V}^{*}}{S_{V}}\right)\left[\mu_{V}\left(1-S_{V}\right)-\mu_{V} \epsilon_{2} I_{V}-\beta_{1} \theta_{2} S_{V} I_{H}\right] \\
& +\omega_{6}\left(\frac{I_{V}-I_{V}^{*}}{I_{V}}\right)\left[\beta_{1} \theta_{2} S_{V} I_{H}-\mu_{V}\left(1-\epsilon_{2}\right) I_{V}\right] .
\end{aligned}
$$

Now, let

$$
\phi_{1}=\mu_{H} \epsilon_{1} I_{H}+\left(\beta_{1} \theta_{1} \alpha_{1} I_{V}+\kappa \beta_{2} \theta_{1} \alpha_{2} I_{U}+\lambda_{1} I_{H}\right) S_{H}
$$

and

$$
\phi_{2}=\mu_{V} \epsilon_{2} I_{V}+\beta_{1} \theta_{2} S_{V} I_{H}
$$

and choose

$$
\omega_{3}=\omega_{4}=\mu_{V} \epsilon_{2} I_{V}^{*}+\beta_{1} \theta_{2} S_{V}^{*} I_{H}^{*}
$$

and

$$
\omega_{5}=\omega_{6}=\mu_{H} \epsilon_{1} I_{H}^{*}+\left(\beta_{1} \theta_{1} \alpha_{1} I_{V}^{*}+\kappa \beta_{2} \theta_{1} \alpha_{2} I_{U}^{*}+\lambda_{1} I_{H}^{*}\right) S_{H}^{*}
$$

then, using the fact that $S_{H}^{\prime}=0, I_{H}^{\prime}=0 S_{U}^{\prime}=0, I_{U}^{\prime}=0$ at the AE, we can write the 
following:

$$
\mu_{H}=\omega_{6}+\mu_{H} S_{H}^{*}, \quad\left(\gamma_{1}+\mu_{H}\right) I_{H}^{*}=\omega_{6}, \quad \mu_{U}=\omega_{4}+\mu_{U} S_{U}^{*}
$$

Using the above relations to rewrite $\mathcal{L}_{2}^{\prime}(t)$, we have

$$
\begin{aligned}
\mathcal{L}_{2}^{\prime}(t) & =\omega_{4}\left(1-\frac{S_{H}^{*}}{S_{H}}\right)\left[\omega_{6}-\mu_{H}\left(S_{H}-S_{H}^{*}\right)-\phi_{1}\right]+\omega_{4}\left(1-\frac{I_{H}^{*}}{I_{H}}\right)\left[\phi_{1}-\frac{\omega_{6}}{I_{H}^{*}} I_{H}\right] \\
& +\omega_{6}\left(1-\frac{S_{V}^{*}}{S_{V}}\right)\left[\omega_{4}-\mu_{V}\left(S_{V}-S_{V}^{*}\right)-\phi_{2}\right]+\omega_{6}\left(1-\frac{I_{V}^{*}}{I_{V}}\right)\left[\phi_{2}-\frac{\omega_{4}}{I_{V}^{*}} I_{V}\right] \\
& =-\frac{\omega_{4} \mu_{H}}{S_{H}}\left(S_{H}-S_{H}^{*}\right)^{2}-\frac{\omega_{6} \mu_{V}}{S_{V}}\left(S_{V}-S_{V}^{*}\right)^{2}+\omega_{4} \phi_{1} \frac{S_{H}^{*}}{S_{H}}\left(1-\frac{I_{H}^{*} S_{H}}{I_{H} S_{H}^{*}}\right) \\
& +\omega_{6} \phi_{2} \frac{S_{V}^{*}}{S_{V}}\left(1-\frac{I_{V}^{*} S_{V}}{I_{V} S_{V}^{*}}\right)+\omega_{4} \omega_{6}\left[4-\left(\frac{S_{H}^{*}}{S_{H}}+\frac{I_{H}}{I_{H}^{*}}+\frac{S_{V}^{*}}{S_{V}}+\frac{I_{V}}{I_{V}^{*}}\right)\right] .
\end{aligned}
$$

Hence, $\mathcal{L}_{2}^{\prime}(t) \leq 0$ under the conditions:

$$
\begin{aligned}
& \frac{S_{H}^{*}}{S_{H}}+\frac{I_{H}}{I_{H}^{*}}+\frac{S_{V}^{*}}{S_{V}}+\frac{I_{V}}{I_{V}^{*}} \geqslant 4 \\
& \frac{I_{H}^{*} S_{H}}{I_{H} S_{H}^{*}} \geqslant 1 \text { and } \frac{I_{V}^{*} S_{V}}{I_{V} S_{V}^{*}} \geqslant 1 .
\end{aligned}
$$

Thus, we can deduce that $\mathcal{L}^{\prime}(t)<0$ if $\mathcal{R}_{2}<1$ and conditions in $(23)$ are hold provided that $\mathcal{R}_{1}>1$ which is required for existence of $\mathrm{AE}$ as discussed earlier. Note that $\mathcal{L}^{\prime}(t)=0$ if $S_{H}=S_{H}^{*}, I_{H}=I_{H}^{*}, S_{V}=S_{V}^{*}, I_{V}=S_{V}^{*}$ and $I_{U}=I_{P}=R_{P}=0\left(R_{P}=0, I_{P}=0\right.$ or $\left.R_{P}=0, I_{U}=0\right)$. Therefore, the AE is the largest invariant set in $\left\{X(t) \in \Omega, \mathcal{L}^{\prime}(t)=0\right\}$. Utilizing LaSalle's invariance principle 28], the $\mathrm{AE}$ is an attractive point in $\Omega$ and hence, all solutions of system (2) converge to $\mathcal{Z}^{(1)}$. The global attractivity and local stability of the AE implies that the AE is globally asymptotically stable provided that the above conditions are satisfied. This result is summarized in the following theorem:

Theorem 7 The AE, given by $\mathcal{Z}^{(1)}$, is globally asymptotically stable if $\mathcal{R}_{2}<1, \mathcal{R}_{1}>1$ and conditions in (23) are hold. 


\section{Numerical Analysis}

In this section, numerical simulations are carried out to demonstrate the obtained

theoretical results of ZIKV model (2) and to study the effect of some model parameters

in the disease transmission dynamics. We use the base line value of the parameters as

listed in Table 1 and appropriate initial conditions. The population size ratio

parameters are assumed to be $\alpha_{1}=2, \alpha_{2}=3$, and $\alpha_{3}=2.5$.

\section{1}

\section{Asymptotic Behavior of the Model}

The asymptotic behavior of the model is graphically verified by plotting the solution curve of human and primates populations for the same choice of initial point. Fig 8 shows that the disease is disappeared from all populations when the basic reproduction number is less than unity. Moreover, when the basic reproduction number is greater than unity, the disease either persists in rural area only as illustrated in Fig 9 or persists in both areas after exhibiting oscillatory behavior as illustrated in Fig 10.

Fig 8. Simulation of the model $(2)$ at $\mathcal{R}_{0}=\mathcal{R}_{1}=0.8715$ and $\mathcal{R}_{2}=0.7813$ for humans and primates populations, respectively.

Fig 9. Simulation of the model $(2)$ at $\mathcal{R}_{0}=\mathcal{R}_{1}=1.1938$ and $\mathcal{R}_{2}=0.9417$ for humans and primates populations, respectively.

Fig 10. Simulation of the model $(2)$ at $\mathcal{R}_{0}=\mathcal{R}_{1}=1.3384$ and $\mathcal{R}_{2}=1.3010$ for humans and primates populations, respectively.

\section{Effect of the Fraction of Susceptible Humans Moving from Rural to Forest Areas}

Here, we investigate the effect of varying the fraction of susceptible humans moving from rural to forest areas $\kappa$ into the number of infected human as shown in Fig 11 . It can be seen that increasing the values of this rate leads to an increase in number of infected human population. On the other hand, reducing this rate has the effect of not only reducing the maximum number of infected human, but also delaying the time it takes to reach the maximum number of infection, i.e., it will have the effect of flattening the curve of infected human population. As a result, it is suggested to avoid traveling to 

areas should be targeted by control strategies in order to eliminate the ZIKV outbreak.

Fig 11. Number of infected humans for different values of $\kappa$.

\section{Effect of Varying Biting Rates}

The effect of varying the values of biting rate of rural mosquitoes on humans $\beta_{1}$, biting rate of forest mosquitoes on humans $\beta_{2}$ and biting rate of forest mosquitoes on primates $\beta_{3}$ on the number of infected humans are shown in Fig 12. Clearly, the biting rate of rural mosquitoes on humans $\beta_{1}$ has the highest impact on the disease transmission among the other biting rates $\beta_{2}$ and $\beta_{3}$ which confirm the theoretical results of sensitivity analysis. Obviously, increasing the values of these rates will increase the number of infected human and reducing their values has the effect of flattening the curve of infected human population. The blue curve in the three graphs in Fig 12 corresponds to the case when $\beta_{1}=\beta_{2}=\beta_{3}=0.3$.

Now, comparing the effect of $\beta_{2}$ and $\beta_{3}$, one could easily see from Fig 12 that the indirect impact of $\beta_{3}$ on infected humans is higher than the direct impact of $\beta_{2}$, which is expected since the infectious primates is considered to be the only source of infection for forest mosquitoes and hence the higher the biting rate on primates the more they become infectious to human even with fixed biting rate on human. On the other hand, fixing the biting rate on primates at low values, taken here to be $\beta_{3}=0.3$, the forest mosquitoes will have less impact on infected human even with increasing biting rate on human. This shows that interaction between forest mosquitoes and primates, represented here through $\beta_{3}$, has a significant impact on the spread of ZIKV among human population when humans travel to forest areas from some reasons. Therefore, as mention previously, control strategies should impose restrictions on travel to affected forest areas of ZIKV in order to control the spread of the disease among the human population.

Fig 12. Number of infected humans for different values of $\beta_{1}$ (Top), $\beta_{2}$ (Middle) and $\beta_{3}$ (Bottom). 


\section{Combined Effect of Transmission Probabilities}

Here, we present the effect of transmission probabilities of infection from and to mosquitoes, $\theta_{1}$ and $\theta_{2}$, respectively. Their effect on human and primate populations is illustrated in Figures 13.14. We vary both $\theta_{1}$ and $\theta_{2}$ and fix the values of all other parameters for the two cases (a) $\mathcal{R}_{0}=\mathcal{R}_{1}>\mathcal{R}_{2}$ and (b) $\mathcal{R}_{0}=\mathcal{R}_{2}>\mathcal{R}_{1}$. The values of $\theta_{1}$ and $\theta_{2}$ are chosen based on the critical values of their product $\Theta=\theta_{1} \theta_{2}$. Fig 13 illustrates their effect for the case (a) with critical values $\Theta^{*}=0.0188$ and $\breve{\Theta}=0.0578$, It can be seen that when the combined transmission probability is small, $\left(\Theta=0.01<\Theta^{*}<\breve{\Theta}\right)$, both human and primate populations converge to the DFE and hence the disease dies out from both rural areas and forest areas. When $\left(\Theta^{*}<\Theta=0.0375<\breve{\Theta}\right)$, the human population reaches the endemic steady state after exhibiting oscillations in the susceptible human population, while the primate population goes to the disease free steady state. This agrees with the obtained stability and bifurcation results, i.e., the system converges to the stable AE for this case. Hence, the disease will persist in rural area and will disappear from forest area. For higher combined transmission probability $\Theta^{*}<\breve{\Theta}<\Theta=0.1$, both populations reach the endemic steady state after exhibiting oscillation in the susceptible populations. Hence, the disease will persist in both areas. Fig 14 is devoted for case (b) with the critical

Fig 13. Combined effect of $\theta_{1}$ and $\theta_{2}$ on human and primates populations for the case (a) $\mathcal{R}_{0}=\mathcal{R}_{1}>\mathcal{R}_{2}$.

Fig 14. Combined effect of $\theta_{1}$ and $\theta_{2}$ on human and primate populations for case (b) $\mathcal{R}_{0}=\mathcal{R}_{2}>\mathcal{R}_{1}$.

values of $\Theta$ are $\breve{\Theta}=0.0293$ and $\Theta^{*}=0.0449$. When $\Theta=0.01<\breve{\Theta}<\Theta^{*}$, the system reaches the stable DFE. However, if the combined transmission probability is $\breve{\Theta}<\Theta=0,0375<\Theta^{*}$ or $\breve{\Theta}<\Theta^{*}<\Theta=0.1$, the system reaches the stable EE after exhibiting oscillation behavior as shown in Fig 14 Reaching a stable AE is not feasible in this case which is consistent with bifurcation results since the system undergoes forward bifurcation only as $\Theta$ passes through critical value $\breve{\Theta}$. 


\section{Conclusion}

A mathematical model of ZIKV illness including direct and vertical transmission of human, mosquito vectors and monkey primates has been proposed. Human movement from rural area to forest area has been also considered. The model was studied and fully analyzed to investigate the impact of primates and human mobility from rural area to forest area in the spread of ZIKV. The boundedness of the invariant region and the positivity of the solution were discussed. The basic reproduction number $\mathcal{R}_{0}$ was computed and expressed in terms of two terms $\mathcal{R}_{1}$ and $\mathcal{R}_{2}$, where $\mathcal{R}_{1}$ represents the transmission due to the interaction within human population $\mathcal{R}_{H H}$ and the interaction between humans and vectors in rural areas $\mathcal{R}_{H V}$, whereas $\mathcal{R}_{2}$ represents the transmission due to the interaction within primate population $\mathcal{R}_{P P}$ and the interaction between primates and vectors in forest areas $\mathcal{R}_{P U}$. The disease threshold occurs either whenever $\mathcal{R}_{H H}+\mathcal{R}_{H V}=1$ if $\mathcal{R}_{0}=\mathcal{R}_{1}=1$ or whenever $\mathcal{R}_{P P}+\mathcal{R}_{P U}=1$ if $\mathcal{R}_{0}=\mathcal{R}_{2}=1$. The proposed model were found to have three equilibria: a disease free equilibrium (DFE), which always exists, an axial equilibrium (AE) exists when $\mathcal{R}_{1}>1$ and it describes an endemic in populations living in rural area only and a full endemic equilibrium (EE) describing an endemic in both forest and rural areas, which exists when $\mathcal{R}_{2}>1$. Then, sensitivity analysis of $\mathcal{R}_{0}$ was carried out, which revealed that $\mathcal{R}_{0}$ is sensitive to practically all model parameters, either positively or negatively. However, the most positive influential parameters are the biting rate of rural mosquitoes on humans and the biting rate of rural mosquitoes on primates, while the recovery rate of humans has the most negative impact. The sensitivity analysis results also showed that the transmission probabilities from and to mosquitoes, $\theta_{1}$ and $\theta_{2}$, respectively, are as important as the ratios of population size between vector and human populations, $\alpha_{1}$, and between vector and primates populations, $\alpha_{3}$, in the disease transmission dynamics. The effect of the transmission probabilities has been further investigated in the bifurcation analysis and through numerical simulation. The bifurcation analysis was investigated analytically and graphically for the case when $\mathcal{R}_{0}=\mathcal{R}_{1}>\mathcal{R}_{2}$ and the case when $\mathcal{R}_{0}=\mathcal{R}_{2}>\mathcal{R}_{1}$, taking one of the transmission probabilities as a bifurcation parameter. The feasibility of forward bifurcation were found to depend on the values of both transmission probabilities $\theta_{1}$ and $\theta_{2}$. It was observed that if $\mathcal{R}_{0}=\mathcal{R}_{1}>\mathcal{R}_{2}$, the 
proposed model may have a double forward bifurcation at DFE and AE, respectively, while if $\mathcal{R}_{0}=\mathcal{R}_{2}>\mathcal{R}_{1}$, it may only experience a forward bifurcation at DFE. It was shown that the DFE is globally asymptotically stable whenever $\mathcal{R}_{0}$ is less than unity, the $\mathrm{AE}$ is globally asymptotically stable whenever $\mathcal{R}_{1}>1>\mathcal{R}_{2}$ under certain conditions and the EE is locally asymptotically stable whenever $\mathcal{R}_{2}>1$ since the direction of the bifurcation was found to be forward. The transmission probabilities were found to play a critical role in the obtained stability results. Finally, Numerical simulations were carried out to demonstrate the obtained theoretical results and to study the effect of some model parameters in the disease transmission dynamics. In particular, it was illustrated that the model converges asymptotically to the DFE whenever $\mathcal{R}_{0}<1$, to the $\mathrm{AE}$ whenever $\mathcal{R}_{1}>1>\mathcal{R}_{2}$ and to the EE after exhibiting oscillatory behavior whenever $\mathcal{R}_{2}>1$. It was also illustrated that the interaction between forest mosquitoes and primates has a significant impact on the ZIKV transmission dynamics among human population and that reducing the fraction of susceptible moving to forest areas has the effect of flattening the curve of infected human population. The critical impact of the combined transmission probabilities from and to mosquitoes in the disease dynamics has been also illustrated numerically. In particular, it was shown that for high transmission probabilities, the disease presists in all populations after exhibiting oscillatory behavior.

\section{References}

1. Olawoyin O, Kribs C. Effects of multiple transmission pathways on Zika dynamics. Infectious Disease Modelling. 2018; 3: 331-344.

2. Biswas SK, Ghosh U, Sarkar S. Mathematical model of zika virus dynamics with vector control and sensitivity analysis. Infectious Disease Modelling. 2020; 5: $23-41$.

3. Biswas A, Kodan P, Gupta N, Soneja M, Baruah K, Sharma KK, Meena S. Zika outbreak in India in 2018. Journal of travel medicine. 2020; 27(4): taaa001. 
4. Saiz JC, Vázquez-Calvo A, Blazquez A B, Merino-Ramos T, Escribano-Romero E, Martin-Acebes M A. Zika virus: the latest newcomer. Frontiers in microbiology. 2016; 7: 496 .

5. Rasmussen S A, Jamieson D J. Teratogen update: Zika virus and pregnancy. Birth Defects Res. 2020; 112(15):1139-1149. doi: 10.1002/bdr2.1781.

6. Althouse B M, Vasilakis N, Sall AA, Diallo M, Weaver S C, Hanley K A. Potential for Zika virus to establish a sylvatic transmission cycle in the Americas. PLoS neglected tropical diseases. 2016; 10(12): e0005055.

7. Bueno MG, Martinez N, Abdalla L, Duarte dos Santos C N, Chame M. Animals in the Zika virus life cycle: what to expect from megadiverse Latin American countries. PLoS neglected tropical diseases. 2016;10(12): e0005073.

8. Buechler CR, Bailey AL, Weiler A M, Barry G L, Breitbach ME, Stewart LM, O'Connor D H. Seroprevalence of Zika virus in wild African green monkeys and baboons. Msphere. 2017; 2(2): e00392-16.

9. Best K, Guedj J, Madelain V, de Lamballerie X, Lim SY, Osuna CE, Perelson AS. Zika plasma viral dynamics in nonhuman primates provides insights into early infection and antiviral strategies. Proceedings of the National Academy of Sciences. 2017;114(33): 8847-8852.

10. Al-Maqrashi K, Al-Musalhi F, Elmojtaba IM, Al-Salti N. The Impact of Mobility between Rural Areas and Forests on the Spread of Zika. arXiv preprint arXiv. $2021 ; 2108.11331$.

11. DM Dudley, MT Aliota, et al. A rhesus macaque model of Asian-lineage Zika virus infection. Nature Communications. 2016;7 :12204.

12. CDC. Zika: Transmission and Risks. https://www.cdc.gov/zika/transmission.

13. P Bonaldo, MC, Nielsen Saine K. Zika virus vertical transmission in children with confirmed antenatal exposure. Nature communications. 2020;11(1):1-8.

14. Thangamani, Saravanan and Huang, Jing and Hart, Charles E and Guzman, Hilda and Tesh, Robert B. Vertical Transmission of Zika Virus in Aedes aegypti 
Mosquitoes. The American Journal of Tropical Medicine and Hygiene. 2016; 95(5): 1169-1173.

15. Bonyah E, Okosun KO. Mathematical modeling of Zika virus. Asian Pacific Journal of Tropical Disease. $2016 ; 6(9): 673-679$.

16. Maxian O, Neufeld A, Talis EJ, Childs L M, Blackwood JC. Zika virus dynamics: When does sexual transmission matter?. Epidemics. 2017;21: 48-55.

17. Suparit P, Wiratsudakul A, Modchang C. A mathematical model for Zika virus transmission dynamics with a time-dependent mosquito biting rate. Theoretical Biology and Medical Modelling. 2018; 15(1): 11.

18. Althouse BM, Durbin AP, Hanley KA, Halstead SB, Weaver SC, Cummings DA. Viral kinetics of primary dengue virus infection in non-human primates: a systematic review and individual pooled analysis. Virology. 2014;452: 237-246.

19. Lai Z, Zhou T, Liu S, Zhou J, Xu Y, Gu J, Chen XG. Vertical transmission of zika virus in Aedes albopictus. PLoS neglected tropical diseases. 2020;14(10): e0008776.

20. Van den Driessche P, Watmough J. Reproduction numbers and sub-threshold endemic equilibria for compartmental models of disease transmission. Mathematical biosciences. 2002;180(1-2): 29-48.

21. Rodrigues HS, Monteiro MTT, Torres DF. Sensitivity analysis in a dengue epidemiological model. Hindawi. In Conference Papers in Science: (Vol. 2013).

22. Tesla B, Demakovsky LR, Packiam HS, Mordecai EA, Rodriguez AD, Bonds MH, et al. Estimating the effects of variation in viremia on mosquito susceptibility, infectiousness, and R0 of Zika in Aedes aegypti. PLoS Negl Trop Dis. 2018; 12(8): e0006733.

23. Sambariya D K, Prasad R. Routh stability array method based reduced model of single machine infinite bus with power system stabilizer. In International Conference on Emerging Trends in Electrical, Communication and Information Technologies (ICECIT-2012).2012 Dec; pp.27-34. 
24. Chavez CC, Feng Z, Huang W. On the computation of $R_{0}$ and its role on global stability. Mathematical Approaches for Emerging and Re-Emerging Infection Diseases: An Introduction. The IMA Volumes in Mathematics and Its Applications. 2002; 125: 31-65.

25. Curtiss D R. Recent extentions of Descartes' rule of signs. Annals of Mathematics. 1918; 251-278.

26. A Dhooge, W Govaerts, YA Kuznetsov, HG Meijer, B Sautois. New features of the software MatCont for bifurcation analysis of dynamical systems. Math Comput Model Dyn Syst. 2008;14:147-175.

27. Perko L. Differential equations and dynamical systems (Vol. 7). Springer Science. Business Media (2013).

28. LaSalle JP. Stability theory for ordinary differential equations. Journal of Differential Equations.1968; 4(1): 57-65. 


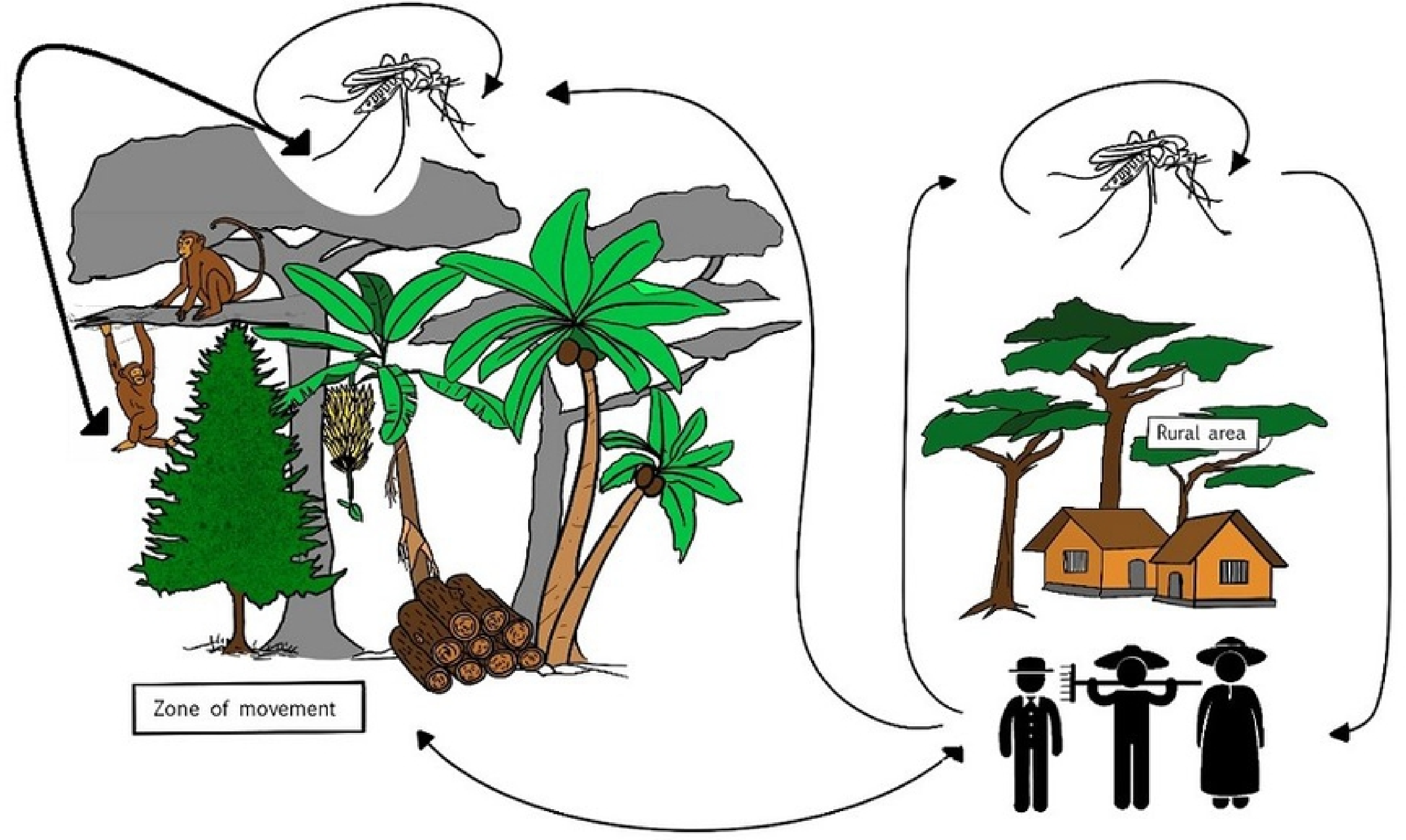

Figure 


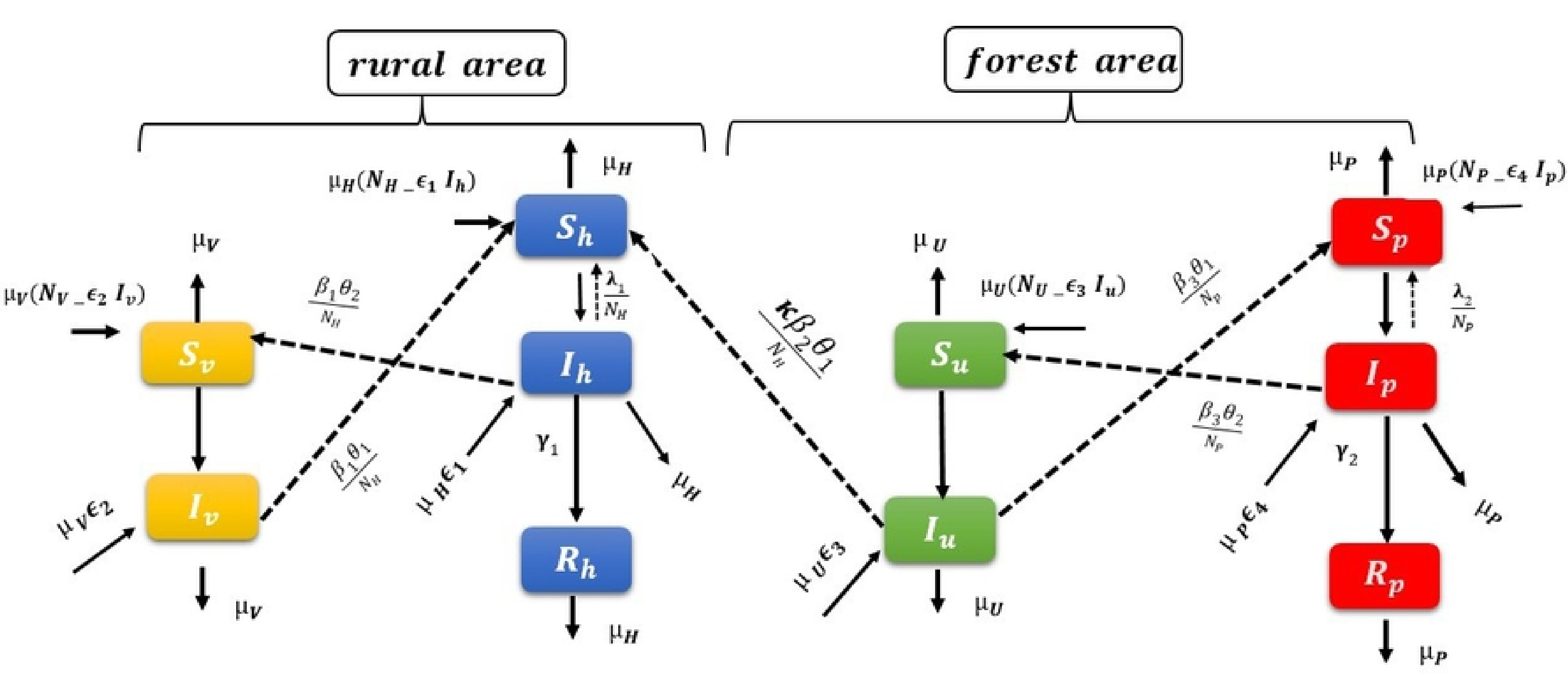

Figure 


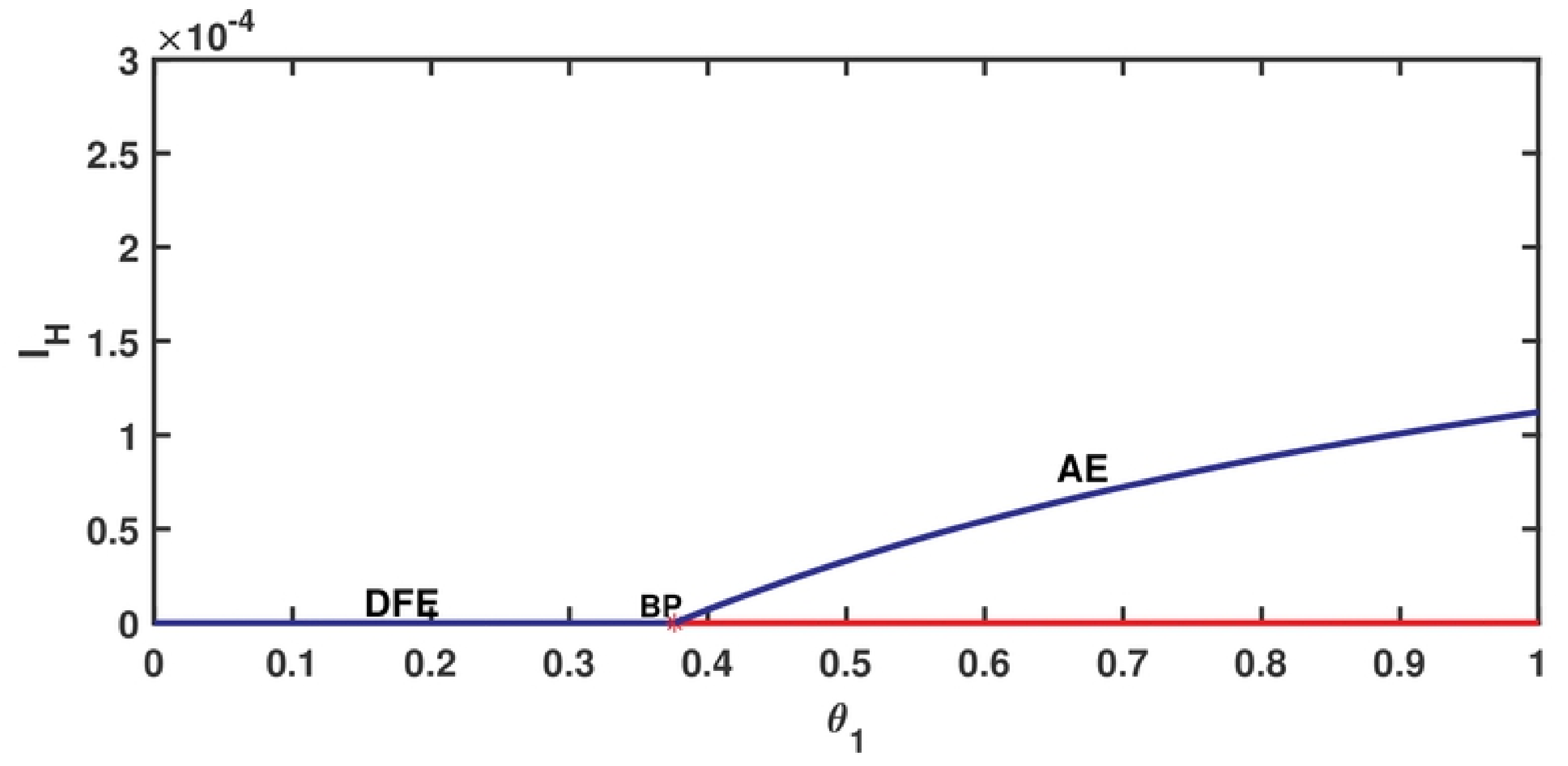

Figure 


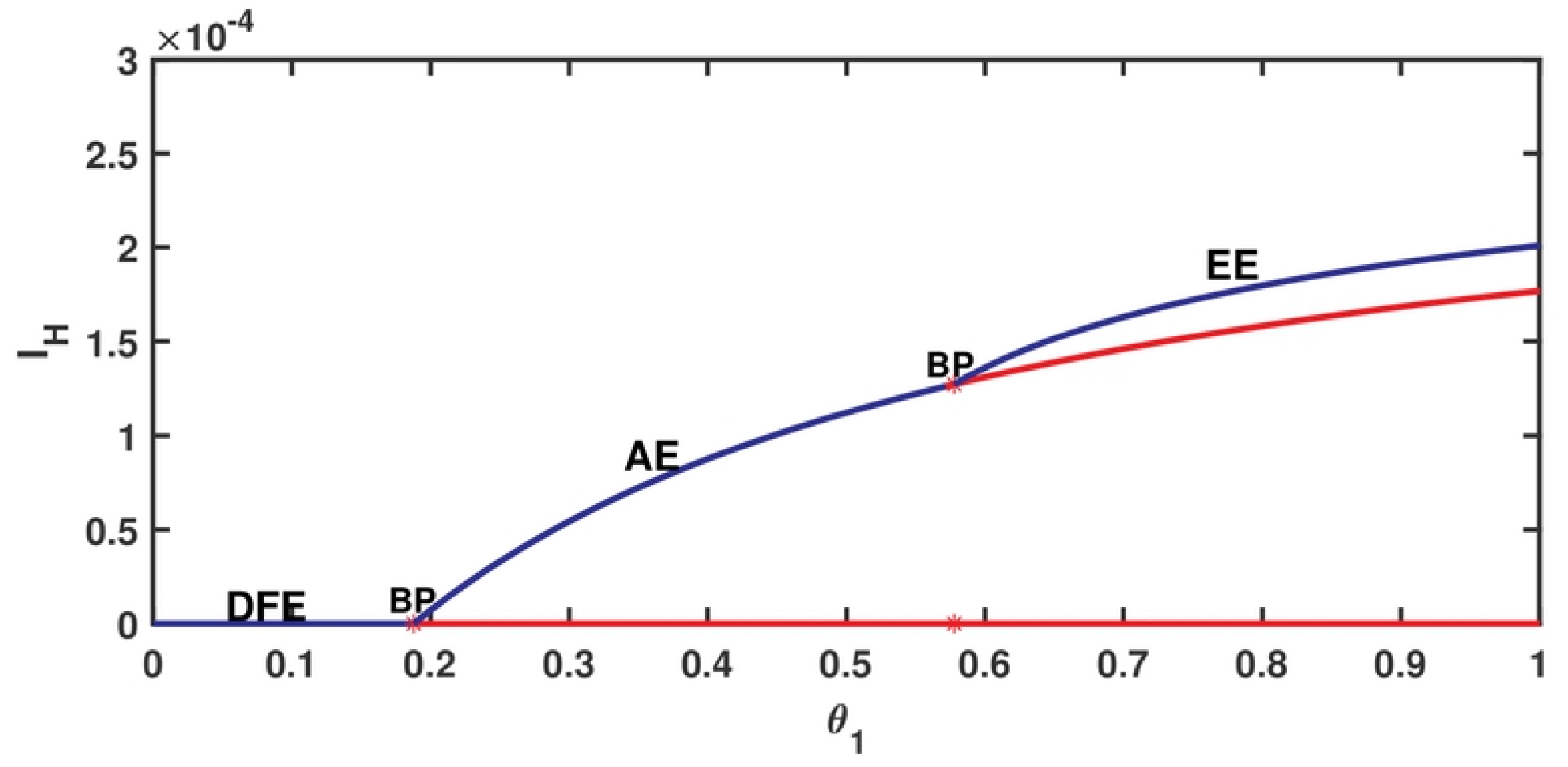

Figure 


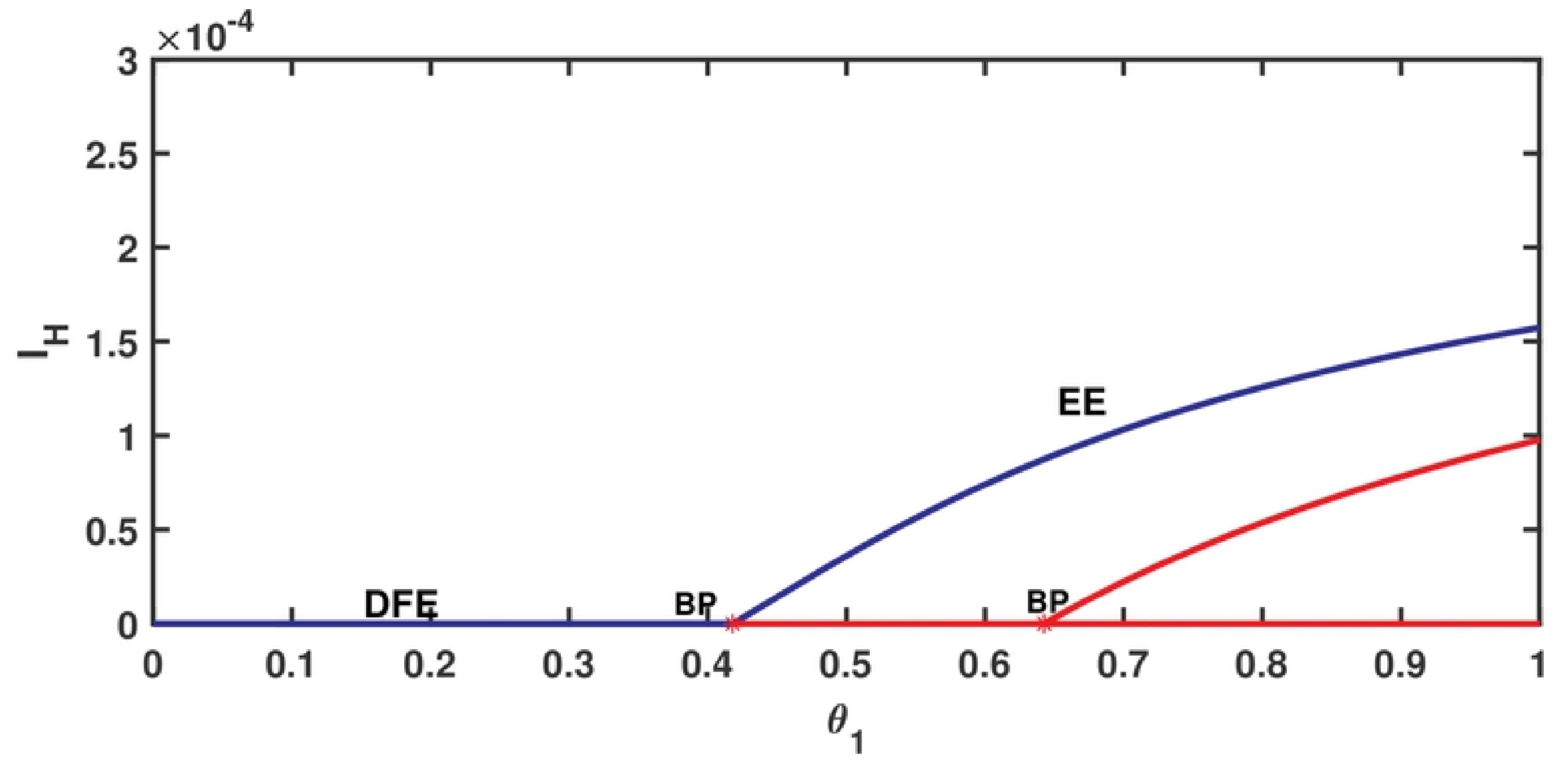

Figure 


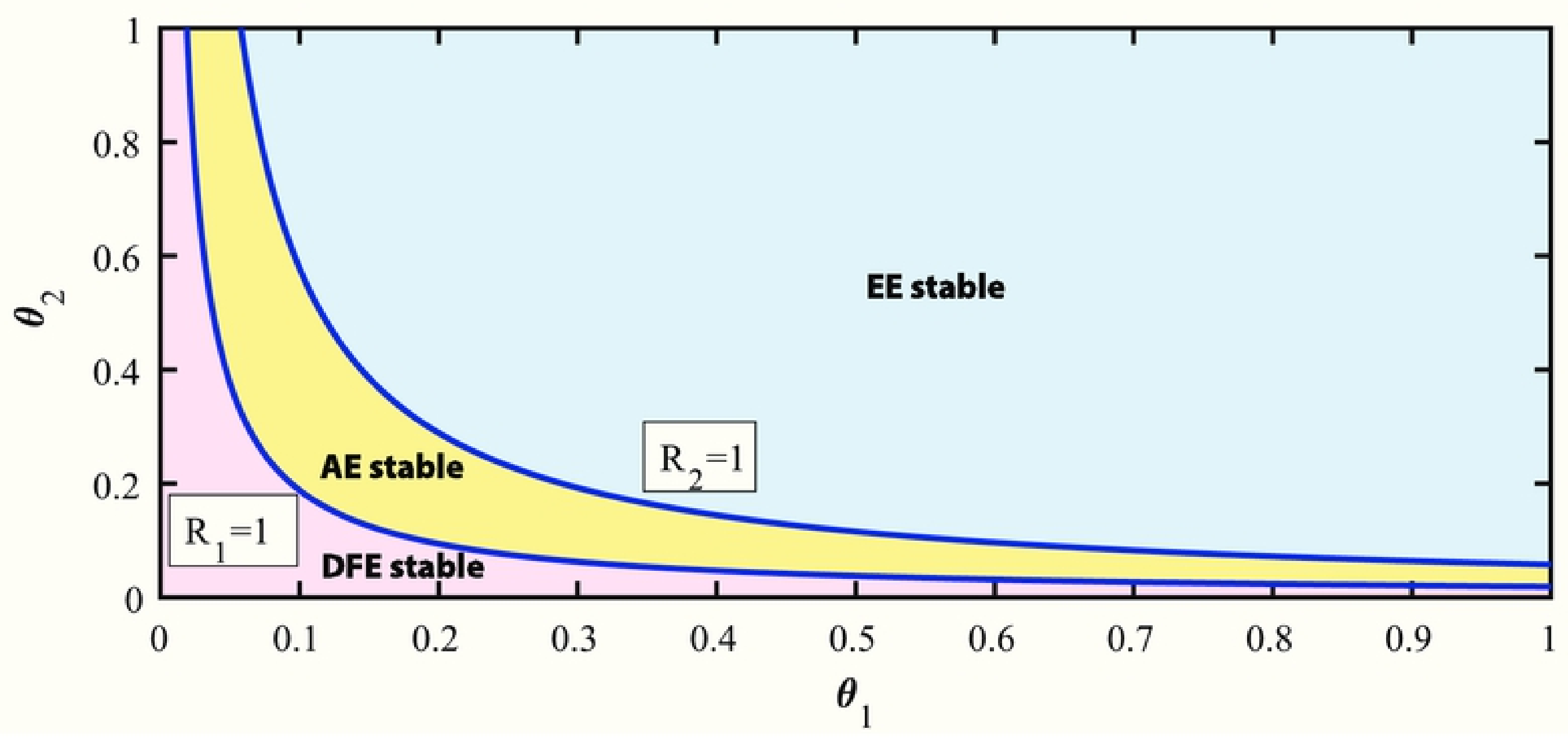

Figure 


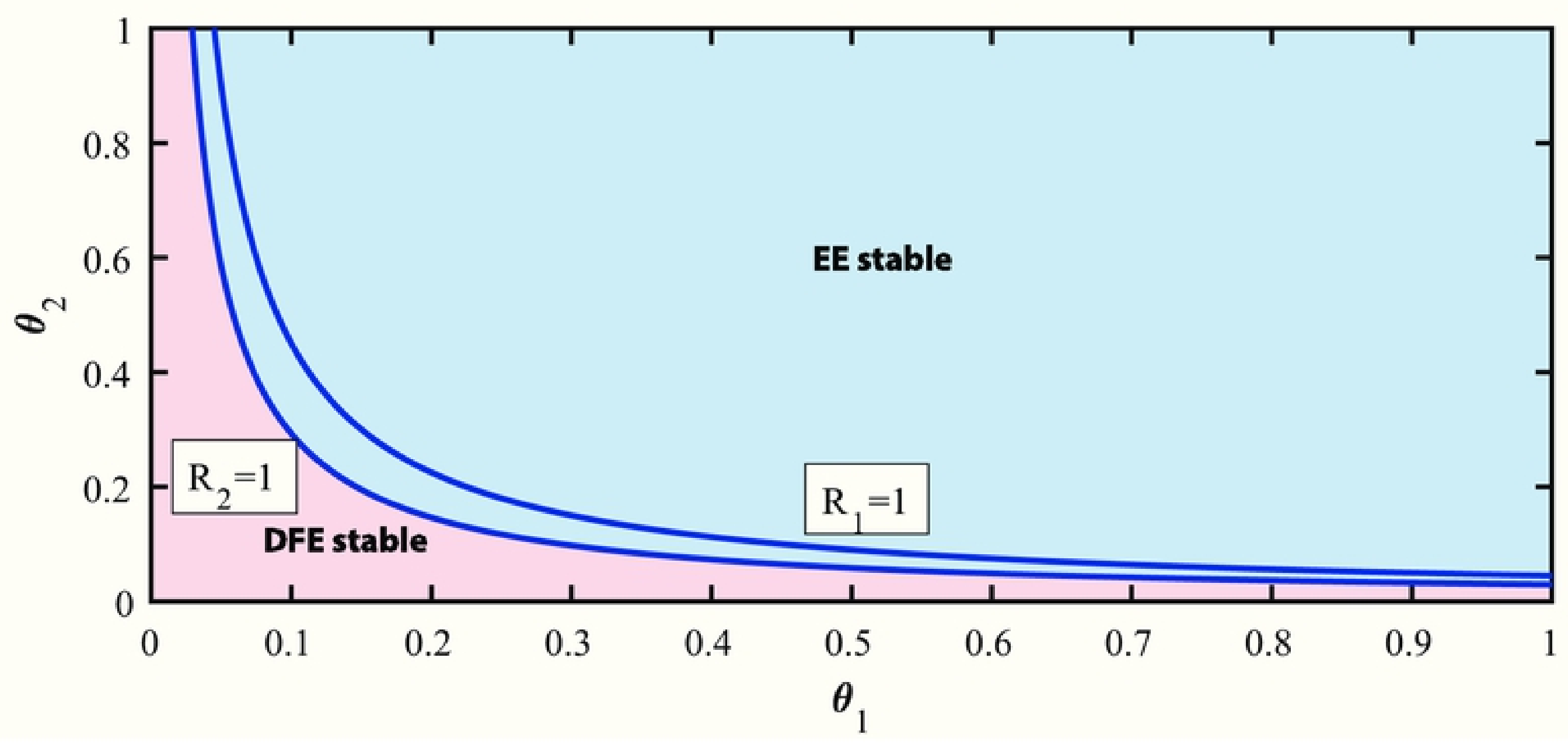

Figure 


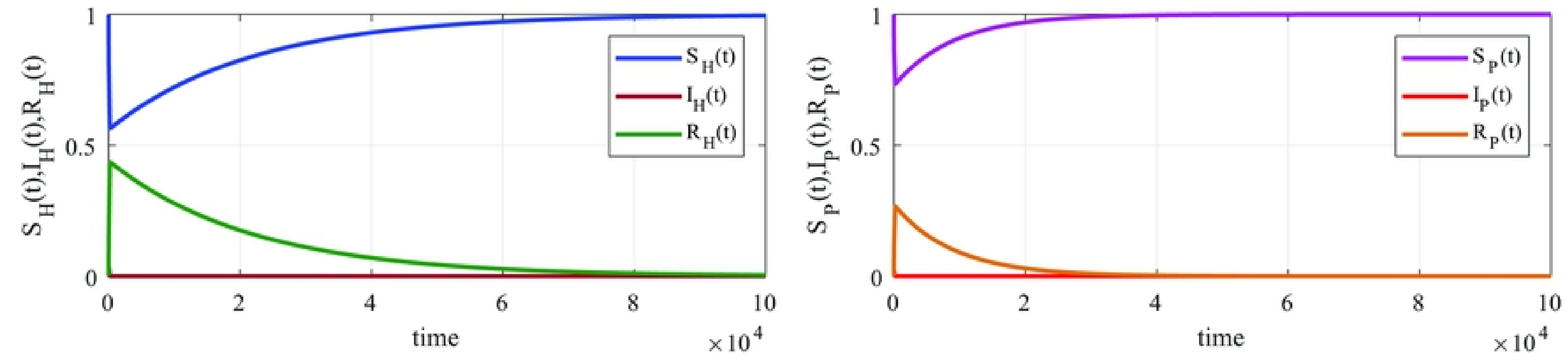

Figure 

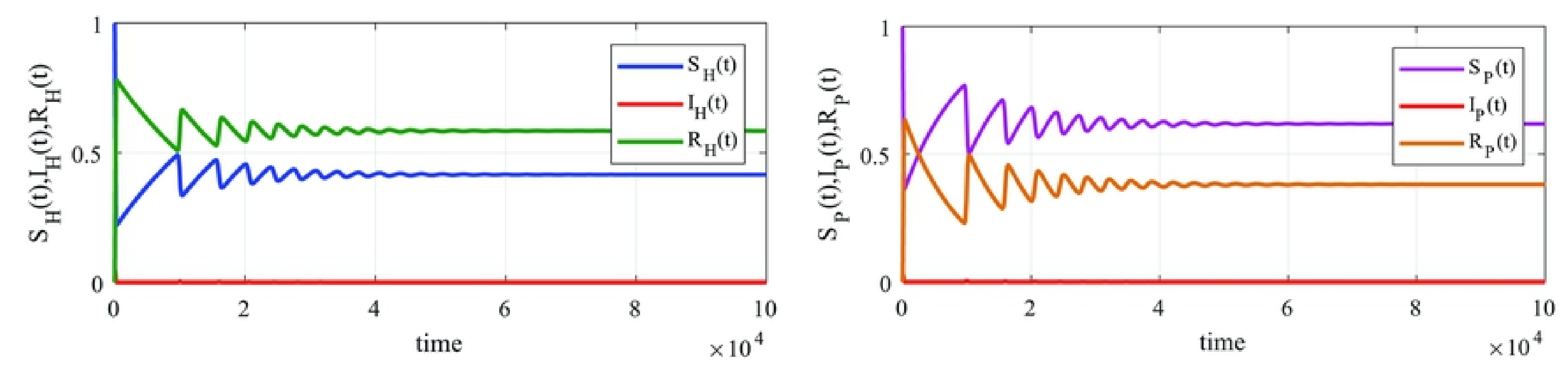

Figure 

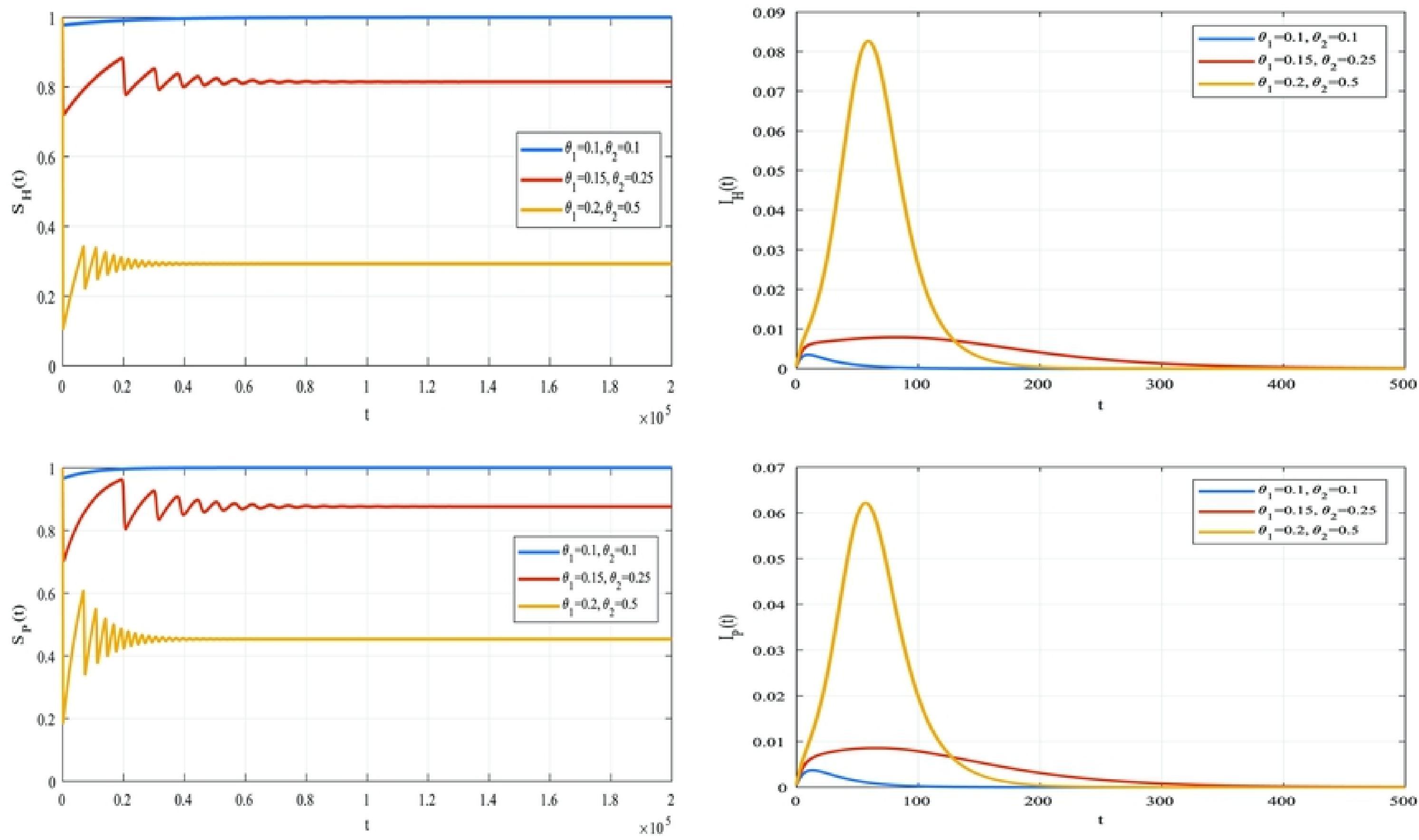

Figure 